\begin{abstract}
EDWARD NICHOLAS SHIPLEY, JUNIOR. Static Aeroelasticity Considerations in Aerodynamic Adaptation of Wings for Low Drag. (Under the direction of Dr. Ashok Gopalarathnam.)

This thesis presents a methodology to calculate the flap angles necessary to reproduce a desired lift distribution for an elastic wing with multiple trailing-edge flaps. The methodology presented builds upon the adaptive wing methodology for rigid wings developed by King and Gopalarathnam. Relevant equations from thin airfoil theory and beam theory are presented, and are then used to develop the solution procedure for the determination of flap angles for an elastic wing. Examples using an elliptic lift distribution are presented, and the effect of wing elasticity on the calculated flap angles and lift distributions is shown. Post-design analysis shows that the flap angles from the current method are successful in achieving an elliptical loading while accounting for using torsional deformations. The methodology is verified by comparing the twist predicted by the methodology to the twist predicted by a finite element analysis. Finally, an example demonstrating how the methodology handles flap effectiveness and flap reversal is provided.
\end{abstract}




\title{
Static Aeroelasticity Considerations in Aerodynamic Adaptation of Wings for Low Drag
}

\author{
by \\ Edward N. Shipley, Jr. \\ A thesis submitted to the Graduate Faculty of \\ North Carolina State University \\ in partial fulfillment of the \\ requirements for the Degree of \\ Master of Science
}

Aerospace Engineering

Raleigh, NC

2006

APPROVED BY:

Dr. Ashok Gopalarathnam

Advisory Committee Chairman

Dr. Pierre A. Gremaude

Advisory Committee Minor Rep.
Dr. Charles E. Hall, Jr.

Advisory Committee Member 


\section{BIOGRAPHY}

Edward N. Shipley, Jr. was born on January 10, 1979 in Holmdel, NJ to Edward N. Shipley, Sr. and Joan E. Shipley. In 1997, he enrolled at Washington University in St. Louis. While at Washington University, he was teaching assistant in the Computer Science department, a co-op at Emerson Motor Company, and a Commodore of the Sailing Club. In May of 2002, he graduated from Washington University with a Bachelor of Science degree in Mechanical Engineering and a minor in Computer Science. In January of 2004, he enrolled at North Carolina State University to earn a Master of Science degree in Aerospace Engineering. A year later, he joined the applied aerodynamics group to research adaptive wing technology under Dr. Ashok Gopalarathnam. He will graduate from North Car-

olina State in May 2006 with a Masters in Aerospace Engineering and a minor in Mathematics. 


\section{ACKNOWLEDGEMENTS}

First of all, I would like to thank Dr. Ashok Gopalarathnam. During the past year, he has guided through my research and taught me all that I know about theoretical aerodynamics.

I also want to thank Dr. Charles Hall and Dr. Pierre Gremaud for agreeing to be members of my thesis committee. As a student in their classrooms, I have learned much from them.

A special thanks goes to my parents, Edward and Joan Shipley, who have always been there, supporting me. To my two sisters, Lisa and Linda, whom I have received much advice from.

Finally, thanks goes my fellow graduate students. They have helped me through many of the challenges of being a graduate student. 


\section{Table of Contents}

List of Tables $\ldots \ldots \ldots \ldots \ldots \ldots \ldots \ldots$ vi

List of Figures $\ldots \ldots \ldots \ldots \ldots \ldots \ldots \ldots$ vii

Nomenclature .................. ix

Chapter 1 Introduction $\ldots \ldots \ldots \ldots \ldots \ldots \ldots$

1.1 Prior Research in Adaptive Wings . . . . . . . . . . . . . . . . 1

1.2 Research Objectives. . . . . . . . . . . . . . . . . 4

1.3 Outline of Thesis $\ldots \ldots \ldots \ldots \ldots \ldots$

Chapter 2 Methodology ................ 6

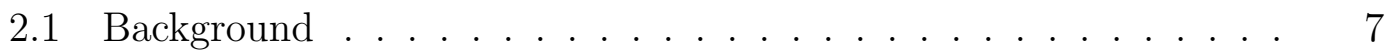

2.1.1 Slender Beam Theory . . . . . . . . . . . . . . . . . . 7

2.1.2 Applied Torque on Wing Sections . . . . . . . . . . . 9

2.1.3 Thin Airfoil Theory to Estimate Flap Effects . . . . . . . . 10

2.1.4 Basic and Additional Lift Distributions . . . . . . . . . 11

2.1.5 Finite Element Analysis Model . . . . . . . . . . . . . 12

2.2 Procedure for Determining Optimum Flap Angles . . . . . . . . . 14

2.3 Verification via Finite Element Analysis _. . . . . . . . . 18

2.4 Summary . . . . . . . . . . . . . . . . . . . . 19 
Chapter 3 Results................... 20

3.1 Elliptical Lift Distribution . . . . . . . . . . . . . . . 22

3.2 Flap Reversal . . . . . . . . . . . . . . . . . . . . . . . . . . . . 29

3.3 Summary . . . . . . . . . . . . . . . . . . . . . . 32

Chapter 4 Conclusion $\ldots \ldots \ldots \ldots \ldots \ldots \ldots$

4.1 Concluding Remarks . . . . . . . . . . . . . . . . 34

4.2 Suggestions for Future Research . . . . . . . . . . . . . 35

Chapter 5 References................. 37 


\section{List of Tables}

3.1 Geometry of the model wing . . . . . . . . . . . . . 20

3.2 Material properties of aluminum alloy . . . . . . . . . 21 


\section{List of Figures}

1.1 AFTI F-111 modified with the Mission Adaptive Wings (Courtesy of NASA Dryden Research Center). . . . . . . . . . . . . . . 2

1.2 F/A-18A test aircraft for the Active Aeroelastic Wing project (Courtesy of NASA Dryden Research Center). . . . . . . . . . . 3

2.1 Illustration of the half span of a wing with 5 flaps $(m)$ and 20 spanwise locations $(n) \ldots \ldots . \ldots 6$

2.2 Three cell thin-walled shell. . . . . . . . . . . . . . . . . 7

2.3 Locations of the aerodynamic center, elastic axis, and center of gravity for a typical wing section. . . . . . . . . . . . . 10

2.4 Basic and additional $C_{l}$ distributions for a wing with a flap deflected $1 \mathrm{deg}$ and $5 \mathrm{deg}$ of wash-out. . . . . . . . . . . . . 13

2.5 Comparison of $C_{l}$ distributions calculated directly and via superposition. . . . . . . . . . . . . . . . . 13

3.1 Planview of example wing (RHS shown). . . . . . . . . . . . . 21

3.2 Example cross section of the wing structure. . . . . . . . . . . . 21

3.3 Comparison of the diagonal elements of $C^{\theta \theta}$ for the stiff wing. The results from FEA and from the multi-cell torsional-rigidity estimation methodology described in Sec. 2.1.1 are plotted. . . . . . . .

3.4 Comparison of the diagonal elements of $C^{\theta \theta}$ for the flexible wing. The results from FEA and from the multi-cell torsional-rigidity estimation methodology described in Sec. 2.1.1 are plotted. . . . .

3.5 Chord-wise location of the elastic axis for the stiff wing as calculated using results from FEA. . . . . . . . . . . . . . . . .

3.6 Chord-wise location of the elastic axis for the flexible wing as calculated using results from FEA. . . . . . . . . . . . . . .

3.7 Comparison of flap angles for the stiff wing calculated using an elastic wing assumption and a rigid wing assumption for $C_{L}$ of 0.1 , 0.5, and 0.8. . . . . . . . . . . . . . . . .

3.8 Comparison of flap angles for the flexible wing calculated using an elastic wing assumption and a rigid wing assumption for $C_{L}$ of 0.1 , 0.5 , and $0.8 \ldots \ldots \ldots \ldots \ldots \ldots$ 
3.9 Comparison of $C_{l}$ distributions for the stiff wing. The $C_{l}$ distributions are calculated for an elastic wing with flap angles from (i) the elastic-wing methodology and (ii) the rigid-wing method and compared with the optimum $C_{l}$ distribution. . . . . . . . . .

3.10 Comparison of $C_{l}$ distributions for the flexible wing. The $C_{l}$ distributions are calculated for an elastic wing with flap angles from (i) the elastic-wing methodology and (ii) the rigid-wing method and compared with the optimum $C_{l}$ distribution. . . . . . . . . .

3.11 Comparison of $C_{l}$ distribution from the current method (Eq. 2.31) with that from post-design analysis using the WINGS code. . . .

$3.12 C_{l}$ distributions, flap angles, and drag polars for $C_{L}$ of $0.1,0.5$, and 0.8 for the stiff wing. . . . . . . . . . . . . .

$3.13 C_{l}$ distributions, flap angles, and drag polars for $C_{L}$ of $0.1,0.5$, and 0.8 for the flexible wing. . . . . . . . . . . .

3.14 Wing twist predicted by current methodology compared to the twist calculated via FEA for $C_{L}$ of $0.1,0.5$, and $0.8 \ldots \ldots \ldots$

3.15 Wing twist predicted by current methodology compared to the twist calculated via FEA for $C_{L}$ of $0.1,0.5$, and $0.8 \ldots \ldots 30$

3.16 Flap angles for $C_{L}$ of 0.5 at increasing loads. . . . . . . . 31

$3.17 C_{l}$ distributions for $C_{\text {loptimum }}$ and $C_{l_{a}}$ at $C_{L}$ of $0.5 \ldots \ldots \ldots \ldots 32$

3.18 Wing twist for $C_{L}$ of 0.5 at increasing loads. . . . . . . . 33 


\title{
Nomenclature
}

\author{
$A_{i} \quad$ area of cell $i$ \\ $a_{i j} \quad$ line integral between cell $i$ and cell $j$ \\ $A_{n} \quad$ Fourier series coefficients \\ c local chord length \\ $C_{L} \quad$ wing lift coefficient \\ $C_{l} \quad$ section lift coefficient \\ $C_{l_{a, 1}}$ additional lift coefficient distribution calculated at $C_{L}=1$ \\ $C_{l_{b}} \quad$ basic lift coefficient distribution \\ $C_{m} \quad$ airfoil pitching moment coefficient \\ $C^{\theta \theta} \quad$ torsional influence coefficient matrix \\ F $\quad$ shear stress \\ G shear modulus \\ $g \quad$ acceleration due to gravity \\ I identity matrix \\ $J \quad$ torsional constant
}


$m \quad$ local mass of the wing

$m$ number of flap on the half span of the wing

$N \quad$ load factor

$n$ number of spanwise locations on the half span of the wing

$q \quad$ dynamic pressure

Re Reynolds number

$s \quad$ arc length

$t \quad$ material thickness

$V_{\infty} \quad$ freestream velocity

$x \quad$ distance from leading edge of an airfoil

y spanwise coordinate

$z \quad$ coordinate normal to the chord line

$\alpha \quad$ angle of attack

$\delta_{f} \quad$ flap deflection, positive down

$\eta \quad$ spanwise coordinate

$\Gamma \quad$ vorticity along the wing span

$\gamma \quad$ vorticity along the camberline of an airfoil

$\lambda \quad$ dummy integration variable

$\nu \quad$ kinematic viscosity 
$\rho \quad$ density of air

$\tau \quad$ torque

$\theta \quad$ distance from leading edge of the airfoil in angular coordinates

$\theta \quad$ wing twist due to torsion

\section{Subscripts}

ac aerodynamic center of an airfoil

$c g \quad$ center of gravity

ea elastic axis of the wing 


\section{Chapter 1}

\section{Introduction}

\subsection{Prior Research in Adaptive Wings}

Adaptive wings are generating considerable interest in the US ${ }^{1-3}$ and Europe $e^{4-6}$ because of their potential for drag reduction in 1-g flight and wing load alleviation during maneuvering flight. Recent research at NC State in adaptive wing

technology ${ }^{7,8}$ has shown that a series of flaps placed along the trailing edge of a wing can be used to optimize the wing across several flight conditions. Without these flaps, wings can only be optimized for flight at one particular condition and suffer deficiencies while flying at other conditions. Being able to automatically and dynamically optimize a wing for any flight condition will result in less drag and reduced vehicle weight. With lower drag and weight, the vehicle's maximum velocity, altitude, and range will be increased.

Prior research by King and Gopalarathnam ${ }^{7}$ has resulted in a semi-analytical approach for the determination of optimum flap angles for simultaneously minimizing both induced and profile drag on a laminar-flow wing. The method used a combination of three well-known elements in applied aerodynamics: (i) an approach for determining minimum-induced-drag loadings based on the work of R. T. Jones, ${ }^{9}$ (ii) a method for determining the $C_{l}$-shift in the low-drag range (drag bucket) of laminar airfoils, and (iii) the concept of basic and additional 
lift distributions. Using these elements, the following system of equations, was derived, that when solved, provides the optimum flap angles:

$$
C_{L}\left\{C_{l_{a, 1}}\right\}+\left\{C_{l_{b, t w i s t}}\right\}+\left\{C_{l_{b, \text { camber }}}\right\}+\left[C_{l_{b, f}}\right]\left\{\delta_{f}\right\}=\left\{C_{l_{\text {desired }}}\right\}
$$

Flight testing at NASA has been done in both the areas of variable-camber wings $^{10}$ and inclusion of aeroelasticity into flight control laws. ${ }^{11}$ For the Mission Adaptive Wing (MAW) project a F-111 was modified with leading and trailing edge flaps (Fig. 1.1) that had no gaps on the upper surface of the wing. Pressure and boundary layer data on the wing was taken at various dynamic pressures, Mach speeds, and wing sweeps. The results of the project demonstrated that adaptive wings could greatly improve the performance of an aircraft.

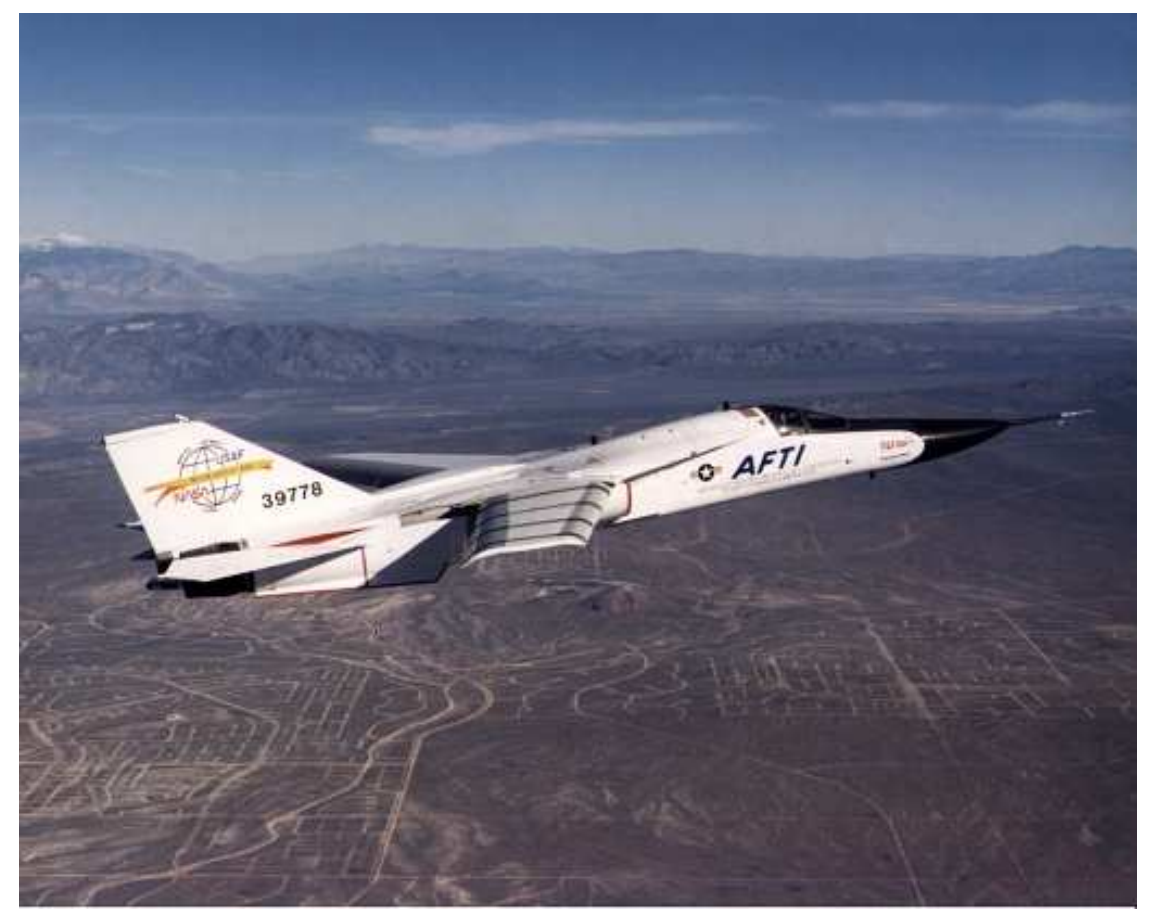

Figure 1.1: AFTI F-111 modified with the Mission Adaptive Wings (Courtesy of NASA Dryden Research Center).

Flight tests of a modified F/A-18 (Fig. 1.2) for the Active Aeroelastic Wing 
(AAW) project at NASA have demonstrated that it is possible to have sufficient roll control on an aircraft with a flexible wing. Previously, engineers have had to reinforce the wing to minimize wing twist in order to maintain the effectiveness of control surfaces in producing a rolling moment. The structures used to reinforce the wing are added weight that could instead be used to increase payload or carry extra fuel to increase range. The test wing for the modified F/A-18 used a more flexible wing than the production F/A-18 and used two leading edge flaps instead of one. With this elastic wing and a flight control system accounting for wing twist, the modified F/A-18 had improved performance in some areas and had nearly the same roll rate as a production $\mathrm{F} / \mathrm{A}-18 .^{12}$

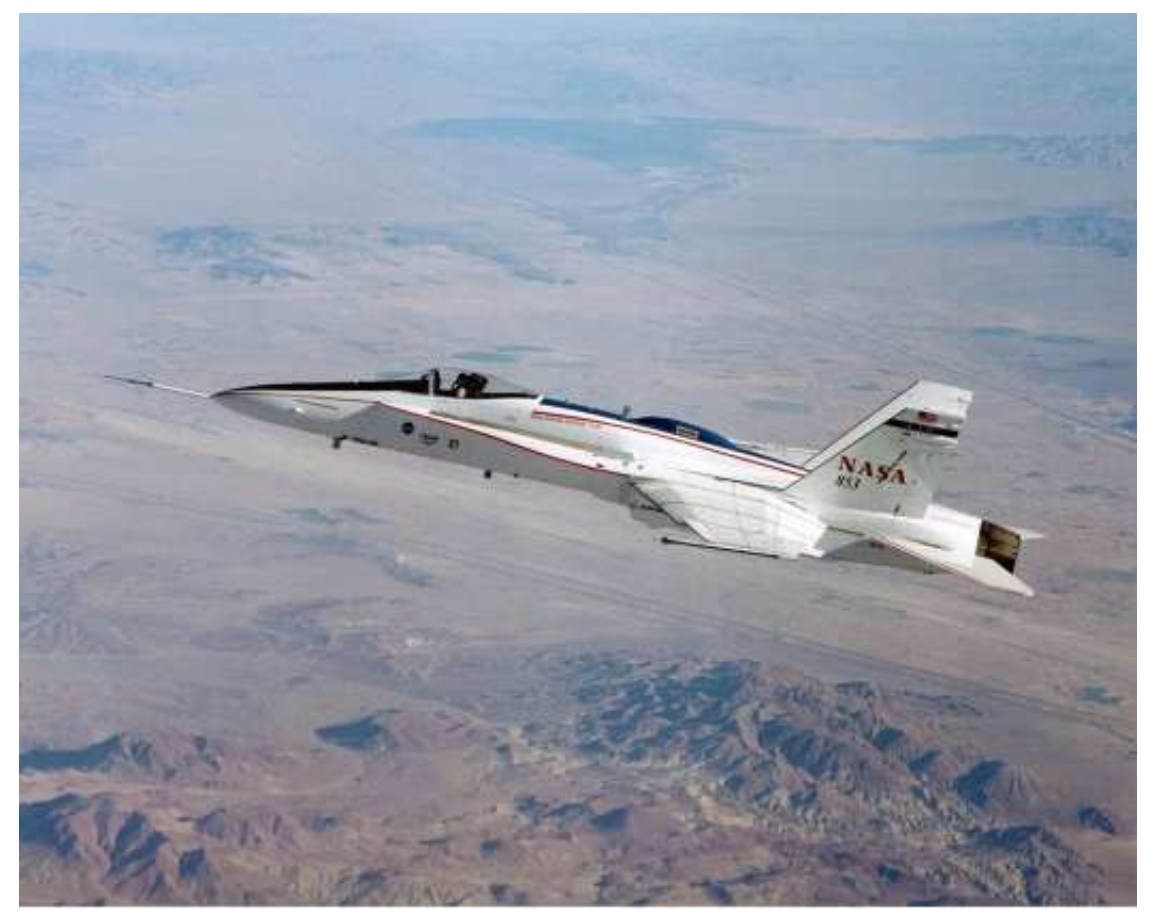

Figure 1.2: F/A-18A test aircraft for the Active Aeroelastic Wing project (Courtesy of NASA Dryden Research Center). 


\section{$1.2 \quad$ Research Objectives}

If the wing is sufficiently rigid, the system of equations developed by King and Gopalarathnam (Eq. 1.1) will allow for an accurate reproduction of a desired lift distribution. However, for applications where low drag is desired, high aspect ratio wings are typically used because of the reduction in induced drag that is achieved with a larger wing span. As the aspect ratio increases, aeroelastic phenomena become more important. Since adaptive flaps will typically be used in conjunction with high aspect ratio to reduce drag, it is important that the system of equations include static aeroelastic effects, especially due to elastic twist.

The goal of this research is to develop a system of equations, similar to the ones written by King and Gopalarathnam, but remove the rigid-wing assumption and include elastic twist of the wing. The results obtained from the equations will be verified by comparison against the results from a finite element analysis (FEA) model.

\subsection{Outline of Thesis}

The following chapter presents the methodology and equations used in this research. It begins with the relevant equations from thin airfoil theory and slender beam theory. The equations from these two theories are combined and added to the rigid-wing methodology of King and Gopalarathnam. This results in a closedform system of equations that accounts for wing twist in the calculation of the flap angles needed to create a desired $C_{l}$ distribution along the wing. Next, the FEA model is presented, and the methodology used to calculate structural properties from FEA is presented. Following is the methodology used to verify the results using FEA.

The next chapter, chapter 3 , will present the results of the methodology of 
chapter 2 when applied to a simple aircraft wing. The results of the current methodology will be compared to the results obtained from the rigid-wing methodology. The effects of elasticity will be shown by comparing results from a more flexible wing. The current methodology will be verified by comparison of the results to those obtained from FEA. Finally, an example demonstrating that the current methodology accounts for flap effectiveness and flap reversal will be presented.

The fourth chapter will summarize the results presented in chapter 3 and how the current methodology advances adaptive wing technology. Areas where more research is needed will mentioned along with their importance towards implementing adaptive wing technology on an aircraft. 


\section{Chapter 2}

\section{Methodology}

For most applications there will be symmetry across the wing span, so it is only necessary to perform the analysis for one half of the span, while including the effect of the other half using symmetry considerations.

The model used for the half-span consists of $m$ flaps and $n$ spanwise locations like those shown in Fig. 2.1. For each spanwise location, there will be an equation relating the lift distribution, torsional moments, elastic twist, and flap deflection. Although all examples that are presented in the following chapter use equal spacing for the flaps and spanwise locations, the equations that are derived do not assume an equal spacing of the flaps or spanwise locations.

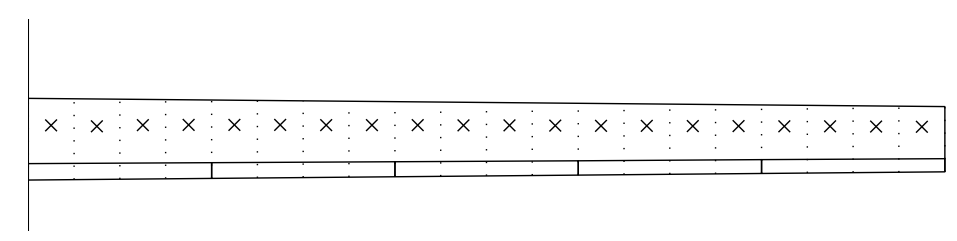

Figure 2.1: Illustration of the half span of a wing with 5 flaps $(m)$ and 20 spanwise locations $(n)$. 


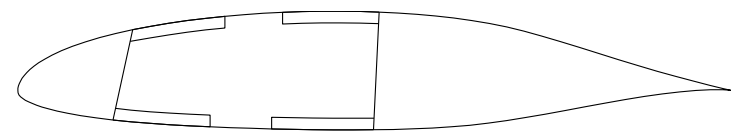

Figure 2.2: Three cell thin-walled shell.

\subsection{Background}

\subsubsection{Slender Beam Theory}

In our analysis of an elastic wing, we will be considering only the elastic twist of the wing. The wing itself is assumed to be unswept and of medium to very high aspect ratio, so that there are no chordwise deformations, and twist and bending deformations are not coupled. Wing bending deformation has also been ignored in the development of the closed-form equations for determining the optimum flap angles, since bending does not contribute significantly to changes in the wing section angle of attack. This allows for the elasticity of the wing to be modeled as a simple torque tube.

The structure of the wing used consists of two interior webs and four spar caps at the junction of the skin and webs. This creates a three-cell thin-walled shell (Fig. 2.2). Normally the flap is disconnected from the wing box, and does not add any structural rigidity to the wing. However, in order to simplify the finite element analysis (FEA) modeling, the outline of the airfoil is modeled, for structural purposes, as a continuous shell with no flap.

According to Bisplinghoff et al., ${ }^{13}$ the relationship between twist and torque for a beam is given by Eq. 2.1:

$$
\{\theta\}=\left[C^{\theta \theta}\right]\{\tau\}
$$

Both $\{\theta\}$ and $\{\tau\}$ are vectors with $n$ elements. $C^{\theta \theta}$ is a square $n \times n$ influence 
coefficient matrix calculated using Eq. 2.2. Each element of this matrix represents the twist caused at spanwise station $y$ due to a unit torque applied at another spanwise location $\eta$.

$$
\begin{aligned}
& C^{\theta \theta}(y, \eta)=\int_{0}^{y} \frac{d \lambda}{G J} \quad(\eta \geq y) \\
& C^{\theta \theta}(y, \eta)=\int_{0}^{\eta} \frac{d \lambda}{G J} \quad(y \geq \eta)
\end{aligned}
$$

In the current work, two approaches are used to determine $C^{\theta \theta}$ : (i) using a FEA model and (ii) using standard methods from aircraft structures to determine the torsional rigidity (GJ) of multi-cell structures. The FEA approach is described later in Sec. 2.1.5. In the following paragraphs, the aircraft structures approach is described.

Before $C^{\theta \theta}$ can be calculated, the torsional stiffness, $G J$, of the wing at every spanwise location needs to be calculated. The torsional constant, $J$, for a three-cell thin-wall structure ${ }^{14}$ is calculated from the system of equations shown in Eq. 2.3. To solve for the torsional constant, the equations are arranged into matrix form as shown in Eq. 2.5 and the relationship between $G \theta, \tau$, and $J$ (Eq. 2.4) is used to make $J$ a variable.

$$
\begin{aligned}
2\left[A_{1} F_{1}+A_{2} F_{2}+A_{3} F_{3}\right] & =\tau \\
\frac{1}{A_{1}}\left[-a_{10} F_{1}+a_{12}\left(F_{2}-F_{1}\right)\right]+2 G \theta & =0 \\
\frac{1}{A_{2}}\left[-a_{20} F_{2}+a_{21}\left(F_{1}-F_{2}\right)+a_{23}\left(F_{3}-F_{2}\right)\right]+2 G \theta & =0 \\
\frac{1}{A_{3}}\left[-a_{30} F_{3}+a_{32}\left(F_{2}-F_{3}\right)\right]+2 G \theta & =0 \\
J=\frac{\tau}{G \theta} &
\end{aligned}
$$




$$
\left[\begin{array}{cccc}
A_{1} & A_{2} & A_{3} & 0 \\
-a_{10}-a_{12} & a_{12} & 0 & 2 A_{1} \\
a_{21} & -a_{20}-a_{21}-a_{23} & a_{23} & 2 A_{2} \\
0 & a_{32} & -a_{30}-a_{32} & 2 A_{3}
\end{array}\right]\left\{\begin{array}{c}
\frac{F_{1}}{\tau} \\
\frac{F_{2}}{\tau} \\
\frac{F_{3}}{\tau} \\
\frac{1}{J}
\end{array}\right\}=\left\{\begin{array}{c}
\frac{1}{2} \\
0 \\
0 \\
0
\end{array}\right\}
$$

The area of each cell, $A_{i}$, and line integrals, $a_{i j}$ are calculated from points located along the surface of the airfoil. $F_{i}$ is the shear stress in the material surrounding $A_{i}$. The line integral (Eq. 2.6) is calculated as the summation of several segments, with each segment being defined by two points. The distance between these two points is calculated and then divided by the thickness of the material for that segment. The line integral of each segment is then summed to give the total line integral between two areas.

$$
a_{i j}=\int \frac{d s}{t}
$$

The area of a cell is calculated by subdividing the cell into several triangles. The area for each triangle is calculated using standard methods.

\subsubsection{Applied Torque on Wing Sections}

It is also necessary to calculate the torque produced at each spanwise location. Generally the elastic axis, aerodynamic center, and center of gravity are at different points along the chord. This causes the lift and the weight of the wing to produce torques in addition to the torque produced by the pitching moment of the airfoil. As illustrated in Fig. 2.3, the torque at a particular spanwise location due to the aerodynamic and inertia loads can be expressed as: 


$$
\tau=q\left(c^{2} C_{m_{a c}}-\left(x_{a c}-x_{e a}\right) c C_{l}\right) d y+N g m\left(x_{c g}-x_{e a}\right)
$$

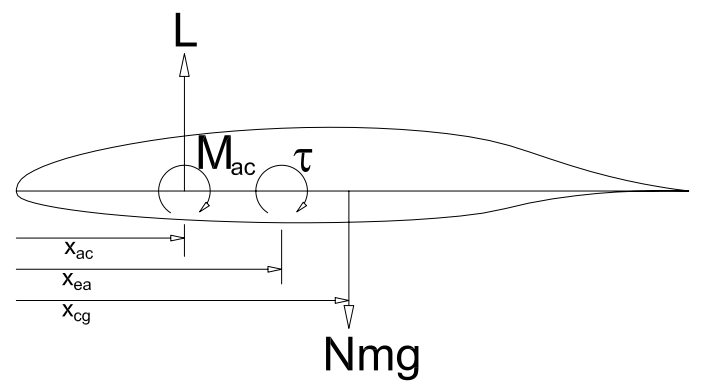

Figure 2.3: Locations of the aerodynamic center, elastic axis, and center of gravity for a typical wing section.

\subsubsection{Thin Airfoil Theory to Estimate Flap Effects}

To minimize the profile drag of a wing, each section of the wing needs to be operating within the low-drag range (LDR) of lift coefficients (drag bucket) of the airfoil. The drag bucket occurs when the stagnation point is at or near the leading edge of the airfoil. Since a stagnation point has zero vorticity, ${ }^{15}$ we can describe the LDR mathematically through thin airfoil theory. We begin by defining $C_{l_{\text {ideal }}}$ as the $C_{l}$ when the vorticity at the leading edge of an airfoil is zero. From thin airfoil theory, the vorticity along the camberline of an airfoil ${ }^{16}$ is given by Eq. 2.8. The Fourier coefficients, $A_{n}$, can be determined as shown in Eqs. 2.9 and 2.10, and $\theta$ is the angular coordinate that corresponds to the chordwise coordinate, $x$, as defined by Eq. 2.11. The lift coefficient can be calculated from the vorticity, and the result of this calculation is shown in Eq. 2.12. For the vorticity at the leading edge to be zero, $A_{0}$ needs to be zero, which results in Eq. 2.13 to calculate $C_{l_{\text {ideal }}}$. Because thin airfoil theory linearizes the aerodynamics, we can determine the incremental effect of a trailing-edge flap deflection on $C_{l_{\text {ideal }}}$ as shown in Eq. 2.14, where $\theta_{f}$ is the angular location, in radians, of the flap hinge location. 


$$
\begin{gathered}
\gamma(\theta)=2 V_{\infty}\left(A_{0} \frac{1+\cos (\theta)}{\sin (\theta)}+\sum_{n=1}^{\infty} A_{n} \sin (n \theta)\right) \\
A_{0}=\alpha-\frac{1}{\pi} \int_{0}^{\pi} \frac{d z}{d x} d \theta_{0} \\
A_{n}=\frac{2}{\pi} \int_{0}^{\pi} \frac{d z}{d x} \cos \left(n \theta_{0}\right) d \theta_{0} \\
\theta=\cos ^{-1}\left(1-2 \frac{x}{c}\right) \\
C_{l}=\pi\left(2 A_{0}+A_{1}\right) \\
C_{l_{\text {ideal }}}=2 \int_{0}^{\pi} \frac{d z}{d x} \cos \left(\theta_{0}\right) d \theta_{0} \\
\Delta C_{l_{\text {ideal }}}=2 \delta_{f} \sin \left(\theta_{f}\right)
\end{gathered}
$$

The pitching moments along the wing are the summation of the airfoil's pitching moment plus the change due to a flap deflection. The change in pitching moment per radian of flap deflection can be predicted by thin airfoil theory ${ }^{17}$ as shown in Eq. 2.15.

$$
C_{m_{\delta_{f}}}=\frac{1}{4} \sin 2 \theta_{f}-\frac{1}{2} \sin \theta_{f}
$$

\subsubsection{Basic and Additional Lift Distributions}

The concept of basic and additional lift distributions takes advantage of the superposition properties of a linear system. The lift (and $C_{l}$ ) distribution is linear when $C_{l}$ vs. $\alpha$ and $C_{l}$ vs. $\Gamma$ are linear relationships. For traditional planar wings at 
subsonic flight conditions where the flow does not separate, both of these relationships are linear. The superposition principle is used to separate the spanwise $C_{l}$ distribution caused by changes in $\alpha, C_{l_{a}}$, from the spanwise $C_{l}$ distribution caused by spanwise changes to the wing geometry, $C_{l_{b}}$. Because $C_{l_{a}}$ is linear we can solve for $C_{l_{a}}$ at $C_{L}=1$, and then scale the result, $C_{l_{a, 1}}$, by $C_{L}$ to get the correct $C_{l_{a}}$ distribution. Also, $C_{l_{b}}$ can be further decomposed into the lift distributions from various sources, such as flap deflections, jig twist, and spanwise changes in section camber.

As as example of superposition, the additional and basic $C_{l}$ distributions for 1-deg flap and 5-deg wash-out are plotted in Fig. 2.4. To find the lift distribution for $5 \mathrm{deg}$ of flap deflection at a $C_{L}$ of $0.4, C_{l_{a, 1}}$ is scaled by 0.4 and $C_{l_{b}}$ for the flap is scaled by 5 . The three $C_{l}$ distributions are then summed. Comparing the $C_{l}$ from superposition to one calculated directly using the vortex-lattice analysis code, WINGS, shows that they match exactly (Fig. 2.5).

The change in lift caused by a flap deflection is calculated using Eq. 2.16. The matrix $C_{l_{b_{f}}}$ can be calculated for a specific wing using Weissinger's method. For this paper, WINGS is used to perform these calculations.

$$
\left\{C_{l_{b, \delta_{f}}}\right\}=\left[C_{l_{\delta_{f}}}\right]\left\{\delta_{f}\right\}
$$

\subsubsection{Finite Element Analysis Model}

A FEA model is used both to calculate $C^{\theta \theta}$ and $X_{e a}$ for use in the solution procedure and also to verify the predicted twist from the solution explained in the following section. The model is constructed in ANSYS using the shell63 element. ${ }^{18}$ The shell63 element is a quadrilateral shell element with an assigned thickness. Shear stress is assumed to vary linearly across the thickness. At the root of the wing a fixed boundary condition is applied to the nodes of the elements, prevent- 


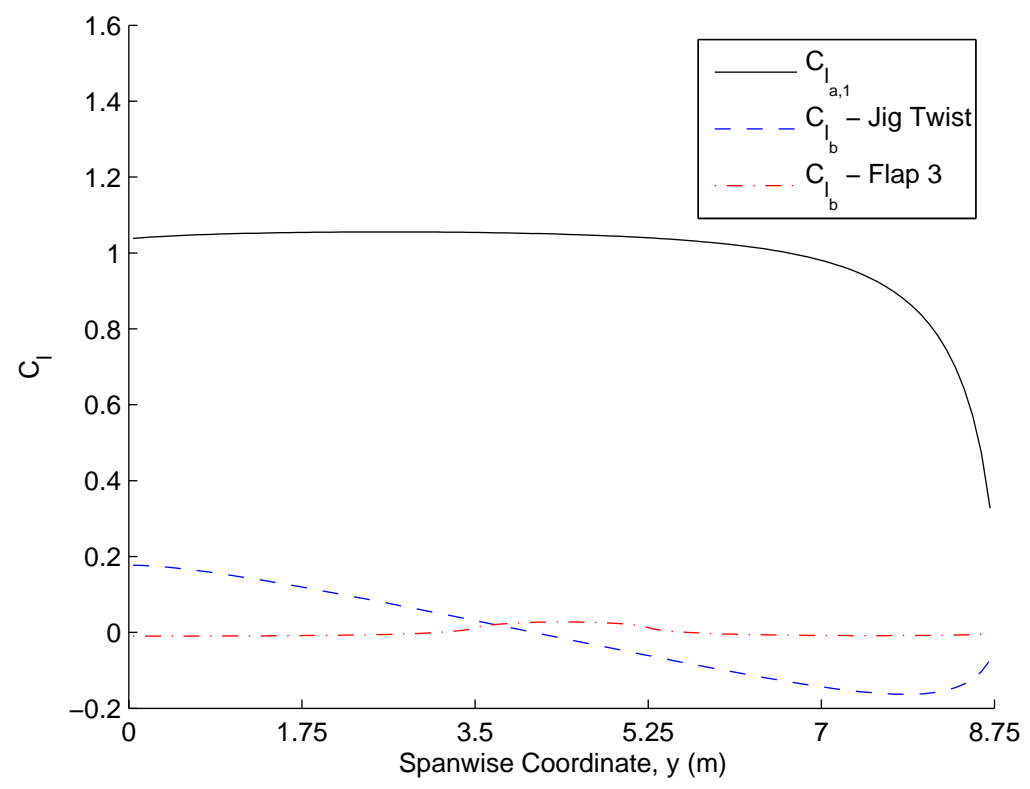

Figure 2.4: Basic and additional $C_{l}$ distributions for a wing with a flap deflected $1 \mathrm{deg}$ and $5 \mathrm{deg}$ of wash-out.

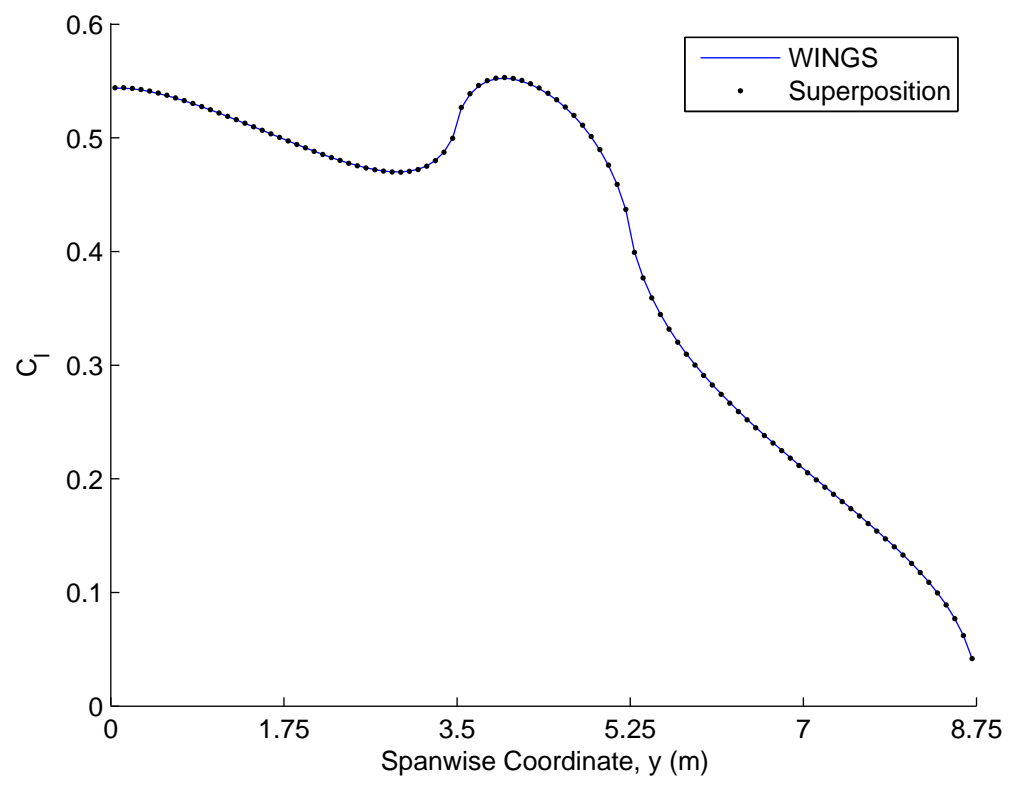

Figure 2.5: Comparison of $C_{l}$ distributions calculated directly and via superposition. 
ing displacement and rotation. Once ANSYS has solved the problem, the twist of the wing at a section is then calculated from the vertical displacement of a node at the leading-edge and a node at the trailing-edge of that section.

To calculate $C^{\theta \theta}$, a torque is created by applying equal vertical point loads, acting in opposite directions, on the upper surface at the leading and trailing webs. For each spanwise location, torque is applied to these nodes and the twist distribution caused by that torque is calculated, creating $n$ sets of twist distributions. Using Eq. 2.1, the elements of $C^{\theta \theta}$ can be calculated from the torques and twist distributions.

$x_{e a}$ is calculated in a similar way to $C^{\theta \theta}$. Instead of a couple, two equivalent forces acting in the same direction are applied on the upper surface to the leading and trailing webs. Using a calculated twist distribution and $C^{\theta \theta}$, a torque is calculated. Since the applied force is known, the moment arm can be calculated from the torque, and then $x_{e a}$ from the moment arm. This procedure is repeated for each spanwise location.

\subsection{Procedure for Determining Optimum Flap}

\section{Angles}

Since the aerodynamics for our system are linear, the $C_{l}$ distribution can be written as the summation of the rigid $C_{l}$ distribution, $C_{l, \text { rigid }}$, and an elastic $C_{l}$ distribution, $C_{l, \text { elastic. }} \quad C_{l, \text { rigid }}$ can be further decomposed into the summation of additional and basic $C_{l}$ distributions as shown in Eq. 2.17. $C_{l, \text { elastic }}$, Eq. 2.18, is the twist of the wing multiplied by $C_{l_{b_{t w i s t}}}$ to give a $C_{l}$ distribution. Each element of $C_{l_{b_{t w i s t}}}$ represents the mean basic $C_{l}$ distribution produced across a flap by a unit twist deflection in the wing at a spanwise location. $C_{l_{b_{t w i s t}}}$ is $m \times n$ in size, so that when it is multiplied by a $n \times 1$ vector the result is a $m \times 1$ vector. This 
matrix along with $C_{l_{a, 1}}, C_{l_{b_{\text {geometric }}}}$, and $C_{l_{b_{\delta_{f}}}}$ can be calculated using WINGS.

$$
\begin{aligned}
\left\{C_{l, \text { rigid }}\right\} & =\left\{C_{l_{a, 1}}\right\} C_{L}+\left\{C_{l_{b_{\text {geometric }}}}\right\}+\left[C_{l_{b_{\delta_{f}}}}\right]\left\{\delta_{f}\right\} \\
\left\{C_{l, \text { elastic }}\right\} & =\left[C_{l_{b_{\text {twist }}}}\right]\{\theta\}
\end{aligned}
$$

Combining Eq. 2.1, 2.7, 2.17, and 2.18 will allow for the calculation of $\delta_{f}, C_{l}$, or $\theta$. To solve for $\delta_{f}$ given a known $C_{l}$ distribution (such as from an elliptical lift distribution), we write the $C_{l}$ distribution as the sum of the $C_{l}$ distribution for the zero-flap condition and the $C_{l}$ distribution with flap deflected to produce the system of equations:

$$
\begin{aligned}
\left\{C_{l_{\text {desired,m }}}\right\}= & \left\{C_{l_{\delta_{0}}}\right\}+\left[C_{l_{\delta_{f}}}\right]\left\{\delta_{f}\right\} \\
\left\{C_{l_{\delta_{0}}}\right\}= & \left\{C_{l_{a, 1}}\right\} C_{L}+\left\{C_{l_{b_{\text {geometric }}}}\right\} \\
& +q\left[C_{l_{b_{\text {twist }}}}\right]\left[C^{\theta \theta}\right]\left(\left\{c^{2} C_{m_{0}} d y\right\}-\left\{\left(x_{a c}-x_{e a}\right) c C_{l_{\text {desired }, n}} d y\right\}\right) \\
& +\left[C_{l_{b_{\text {twist }}}}\right]\left[C^{\theta \theta}\right] N g\left\{m\left(x_{c g}-x_{e a}\right)\right\} \\
{\left[C_{l_{\delta_{f}}}\right]=} & {\left[C_{l_{b_{f}}}\right]+q\left[C_{l_{b_{\text {twist }}}}\right]\left[C^{\theta \theta}\right]\left[c^{2} C_{m_{\delta_{f}}} d y\right] }
\end{aligned}
$$

For the matrix dimensions to match, $C_{l_{\text {desired }}}$ needs to be two different sizes. $C_{l_{\text {desired,n }}}$ is a $n \times 1$ column vector representing the desired $C_{l}$ at each spanwise location. $C_{l_{\text {desired,m }}}$ is a $m \times 1$ column vector representing the $C_{l}$ desired across each flap. $C_{l_{\text {desired,m }}}$ can be calculated from $C_{l_{\text {desired,n }}}$ by taking the mean of the elements associated with each flap.

The matrix $\left[c^{2} C_{m_{\delta_{f}}} d y\right]$ is a diagonal $m \times m$ matrix, and each element of the $m \times m$ matrix is a column vector. Shown below (Eqs. 2.22, 2.23, 2.24, and 2.25) is an example matrix for a wing with 3 flaps per half span and 3 spanwise locations 
per flap. There is one column vector per flap. The elements of the vector are the value of $c^{2} C_{m_{\delta_{f}}} d y$ evaluated for each spanwise location across the flap. If each flap has an equal number of spanwise locations then the column vector will be $(n / m) \times 1$ in size. In the example given with $n=9$ and $m=3$, the column vectors are $3 \times 1$. If the matrix $\left[c^{2} C_{m_{\delta_{f}}} d y\right]$ is expanded, then it will be $n \times m$. When $\left[c^{2} C_{m_{\delta_{f}}} d y\right]$ is multiplied by a vector of flap angles $(m \times 1)$, the result will be a $n \times 1$ vector.

$$
\begin{gathered}
{\left[\begin{array}{ccc}
\left\{c^{2} C_{m_{\delta_{1}}} d y\right\} & 0 & 0 \\
0 & \left\{c^{2} C_{m_{\delta_{f_{2}}}} d y\right\} & 0 \\
0 & 0 & \left\{c^{2} C_{m_{\delta_{f_{3}}}} d y\right\}
\end{array}\right]} \\
\left\{c^{2} C_{m_{\delta_{\delta_{1}}}} d y\right\}=\left\{\begin{array}{c}
c_{1}^{2} C_{m_{\delta_{f_{1,1}}}} d y_{1} \\
\downarrow \\
c_{3}^{2} C_{m_{\delta_{f_{1,3}}}} d y_{3}
\end{array}\right\} \\
\left\{c^{2} C_{m_{\delta_{f_{2}}}} d y\right\}=\left\{\begin{array}{c}
c_{4}^{2} C_{m_{\delta_{\delta_{2,4}}}} d y_{4} \\
\downarrow \\
c_{6}^{2} C_{m_{\delta_{f_{2,6}}}} d y_{6}
\end{array}\right\} \\
\left\{c^{2} C_{m_{\delta_{f_{3}}}} d y\right\}=\left\{\begin{array}{c}
c_{7}^{2} C_{m_{\delta_{\delta_{3,7}}}} d y_{7} \\
\downarrow \\
c_{9}^{2} C_{m_{\delta_{f_{3,9}}}} d y_{9}
\end{array}\right\}
\end{gathered}
$$

Since $C_{l_{\delta_{f}}}$ is singular, no unique solution for $\delta_{f}$ exists. As shown by King and Gopalarathnam, ${ }^{7}$ it is possible to write $\delta_{f}$ as the sum of a mean flap, $\bar{\delta}_{f}$, and a flap variation, $\hat{\delta}_{f}$. This allows us to write $C_{l_{\delta_{f}}}$ as: 


$$
\begin{aligned}
{\left[C_{l_{\delta_{f}}}\right]\left\{\delta_{f}\right\} } & =\left[C_{l_{\hat{\delta}_{f}}}\right]\left\{\hat{\delta}_{f}\right\}+\left[C_{l_{\bar{\delta}_{f}}}\right]\left\{\bar{\delta}_{f}\right\} \\
{\left[C_{l_{\hat{\delta}_{f}}}\right]\left\{\hat{\delta}_{f}\right\} } & =\left\{C_{l_{\text {desired }}}\right\}-\left\{C_{l_{\delta_{0}}}\right\}-\left[C_{l_{\bar{\delta}_{f}}}\right]\left\{\bar{\delta}_{f}\right\} \\
{\left[C_{{l_{\delta_{f}}}}\right] } & =\left[C_{l_{b_{\delta_{f}}}}\right]+q\left[C_{l_{b_{t w i s t}}}\right]\left[C^{\theta \theta}\right]\left[c^{2} C_{m_{\delta_{f}}} d y\right] \\
{\left[C_{l_{\bar{\delta}_{f}}}\right] } & =q\left[C_{l_{b_{t w i s t}}}\right]\left[C^{\theta \theta}\right]\left[c^{2} C_{m_{\delta_{f}}} d y\right]
\end{aligned}
$$

The addition of another equation that prescribes that the mean of $\hat{\delta}_{f}$, weighted by the wing area covered by the flap, is zero allows for the system of equations to be solved for a unique flap variation, $\left\{\hat{\delta}_{f}\right\}$. The mean flap angle is calculated by arranging Eq. 2.14 into the form of Eq. 2.30.

$$
\bar{\delta}_{f}=\frac{C_{L}-C_{l_{\text {ideal }}}}{2 \sin \theta_{f}}
$$

For purposes of verification, being able to calculate the twist and $C_{l}$ distributions for a given set of flap deflections is also of interest. Calculation of the $C_{l}$ distribution for known flap angles can be done rearranging Eq. 2.19 so that only $C_{l}$ terms are on the LHS resulting in Eq. 2.31. The matrix $\left[\left(x_{a c}-x_{e a}\right) c d y\right]$ is a diagonal matrix, so that when multiplied by $\left\{C_{l}\right\}$ the result is the vector in Eq. 2.20.

$$
\begin{aligned}
& \left(I+q\left[C_{l_{b_{t w i s t}}}\right]\left[C^{\theta \theta}\right]\left[\left(x_{a c}-x_{e a}\right) c d y\right]\right)\left\{C_{l}\right\}= \\
& \left\{C_{l_{a, 1}}\right\} C_{L}+\left\{C_{l_{b_{\text {geometry }}}}\right\}+\left[C_{l_{b_{f}}}\right]\left\{\delta_{f}\right\} \\
& +q\left[C_{l_{b_{\text {twist }}}}\right]\left[C^{\theta \theta}\right]\left(\left\{c^{2} C_{m_{0}} d y\right\}+\left[c^{2} C_{m_{\delta_{f}}} d y\right]\left\{\delta_{f}\right\}\right) \\
& +\left[C_{l_{b_{t w i s t}}}\right]\left[C^{\theta \theta}\right] N g\left\{m\left(x_{c g}-x_{e a}\right)\right\}
\end{aligned}
$$

To find the twist distribution, $\{\theta\}$, for a known flap distribution, we substitute Eq. 2.17 and 2.18 into the equation for torque (Eq. 2.7). This produces a rela- 
tionship between $\theta$ and $C_{m}$, which is a function of $\delta_{f}$ :

$$
\begin{gathered}
\left(I+q\left[C^{\theta \theta}\right]\left[\left(x_{a c}-x_{e a}\right) c d y\right]\left[C_{l_{b_{t w i s t}}}\right]\right)\{\theta\}= \\
q\left[C^{\theta \theta}\right]\left(\left\{c^{2} C_{m} d y\right\}-\left[\left(x_{a c}-x_{e a}\right) c d y\right]\left\{C_{l, r i g i d}\right\}\right) \\
+\left[C^{\theta \theta}\right] N g\left\{m\left(x_{c g}-x_{e a}\right)\right\}
\end{gathered}
$$

The twist distribution can now be found by solving the above system of equations.

\subsection{Verification via Finite Element Analysis}

The FEA model is also used for post-design analysis to verify that the solution methodology of Sec. 2.2 is producing the correct results. To verify that the methodology is correct, pressure data from XFOIL is applied to each node of the element to create a linearly varying pressure across the element. To get the pressure data, XFOIL is run at $C_{L}=c C_{l}$ using $R e=V_{\infty} / \nu$ on an airfoil that is scaled

to the correct chord length. ${ }^{19}$ The values for $C_{l}$ are obtained from the lift distribution calculated by the equations in Sec. 2.2. The pressure data is then fitted to the nodes of the FEA model using a linear interpolation. The twist distribution from FEA is then compared to the twist predicted by the equations in Sec. 2.2. Correlation between the two twist distributions will be used to verify the results from the solution procedure. 


\subsection{Summary}

In this chapter, the solution procedure was developed for determining the optimum flap angles for an elastic wing from well-known theories and prior research on the subject by King and Gopalarathnam. In the following chapter, the procedure will be applied to an example wing and the results will be verified by comparison with the results from post-design analysis using FEA. 


\section{Chapter 3}

\section{Results}

To properly demonstrate the effect that elastic twist can have on the flap distribution, a very high aspect ratio flexible wing is used. The example wing used in this chapter has an aspect ratio of 25 , a wing area of $12.25 \mathrm{~m}^{2}$, a taper ratio of 0.8 , and operating at an $\operatorname{Re} \sqrt{C_{l}}$ of $3 \times 10^{6}$. The rest of the wing geometry is listed in table 3.1. Figure 3.1 shows the planview for this wing. There are 5 flaps placed on the trailing edge. Each flap occupies $20 \%$ of the chord in width and one-fifth of the half span of the wing. The wing has zero geometric twist. The weight of the wing is assumed to be concentrated along the elastic axis, so that it will not create a pitching moment and can be ignored.

The airfoil used for the wing is the same airfoil used by King and Gopalarathnam $^{7}$ for their research. At $R e \sqrt{C_{l}}=3 \times 10^{6}$ the airfoil has a low-drag range from $C_{l}$ of 0.3 to 0.7 , and an $\alpha_{Z L}$ of -3.44 deg. The wing box used is shown in Fig. 3.2.

\begin{tabular}{lr}
\hline \hline Parameter & Value \\
\hline Aircraft Weight (W) & $29376 \mathrm{~N}$ \\
Wing Area (S) & $12.25 \mathrm{~m}^{2}$ \\
Wing Aspect Ratio (AR) & 25 \\
Taper Ratio & 0.80 \\
Wing Span (b) & $17.5 \mathrm{~m}$ \\
Mean Chord & $0.7 \mathrm{~m}$ \\
\hline
\end{tabular}

Table 3.1: Geometry of the model wing 
Figure 3.1: Planview of example wing (RHS shown).

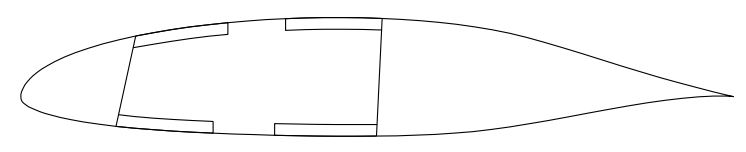

Figure 3.2: Example cross section of the wing structure.

A simple structural cross section was used to allow for the wing to be easily modeled in ANSYS, while retaining all the necessary features to demonstrate the current method. To demonstrate the effect of elasticity, two sets of skin thicknesses are used. For the first wing, the skin, interior webs, and ribs are 0.508 $\mathrm{mm}$ (0.020 in) in thickness. The thickness of the spar caps is $12.7 \mathrm{~mm}$ (0.50 in). For the second wing, the thicknesses of the first wing are scaled by 0.5 . In the following section, the first wing is referred to as the "stiff wing", and the second wing is referred to as the "flexible wing". For both wings, the ribs are a thin solid sheet of metal placed at every other spanwise location so that the skin does not deform. The shear webs are placed at 0.15 chord and 0.50 chord. The material properties used (table 3.2) are typical of aluminum alloys.

\begin{tabular}{lr}
\hline \hline Parameter & Value \\
\hline Young's modulus (E) & $7.24 \times 10^{10} \mathrm{~N} / \mathrm{m}^{2}$ \\
Shear modulus (G) & $2.6 \times 10^{10} \mathrm{~N} / \mathrm{m}^{2}$ \\
Poisson's Ratio & 0.33 \\
\hline
\end{tabular}

Table 3.2: Material properties of aluminum alloy 


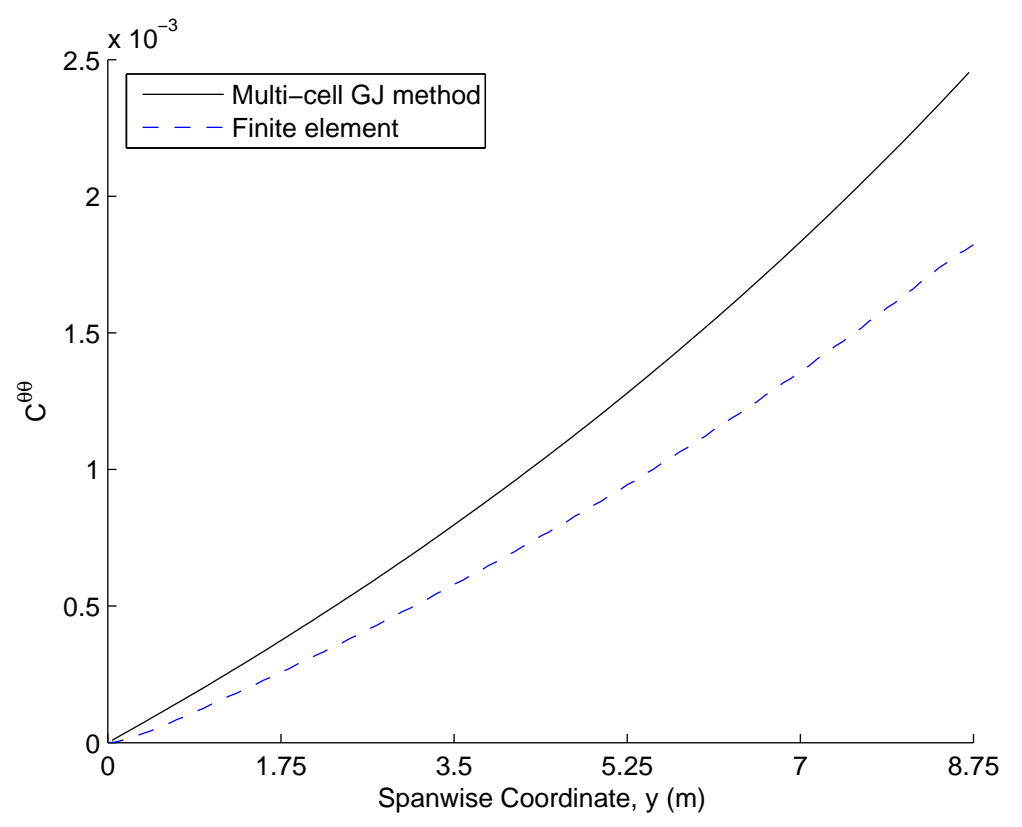

Figure 3.3: Comparison of the diagonal elements of $C^{\theta \theta}$ for the stiff wing. The results from FEA and from the multi-cell torsional-rigidity estimation methodology described in Sec. 2.1.1 are plotted.

\subsection{Elliptical Lift Distribution}

While the equations can be used to reproduce any lift distribution, this example uses the well-known elliptical lift distribution for minimizing induced drag on a planar wing. The corresponding $C_{l}$ distribution is referred to as $C_{l_{\text {optimum }}}(y)$ in this paper. Using the methods presented in chapter 2, the structural properties of the wing $\left(C^{\theta \theta}\right.$ and $\left.X_{e a}\right)$ are calculated using finite element analysis (FEA) as described in Sec. 2.1.5. The results of these calculations are shown in Figs. 3.3, 3.4, 3.5, and 3.6. Comparing the diagonal elements of $C^{\theta \theta}$ from FEA and from the multi-cell GJ methodology from Sec. 2.1 shows a reasonable correlation. The matrix from FEA is used for this example so there will be better agreement when the twist calculated by the current methodology is verified using post-design analysis with FEA.

Using the $C^{\theta \theta}$ matrix and $X_{e a}$ from finite element, optimum flap deflections 


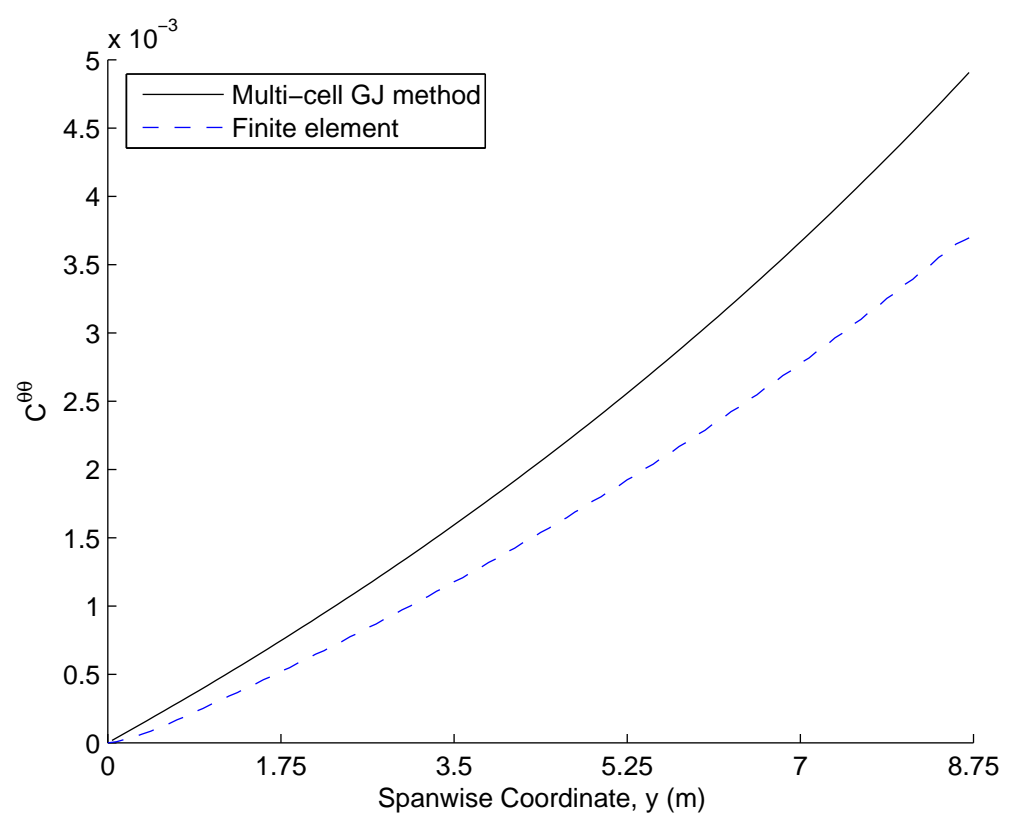

Figure 3.4: Comparison of the diagonal elements of $C^{\theta \theta}$ for the flexible wing. The results from FEA and from the multi-cell torsional-rigidity estimation methodology described in Sec. 2.1.1 are plotted.

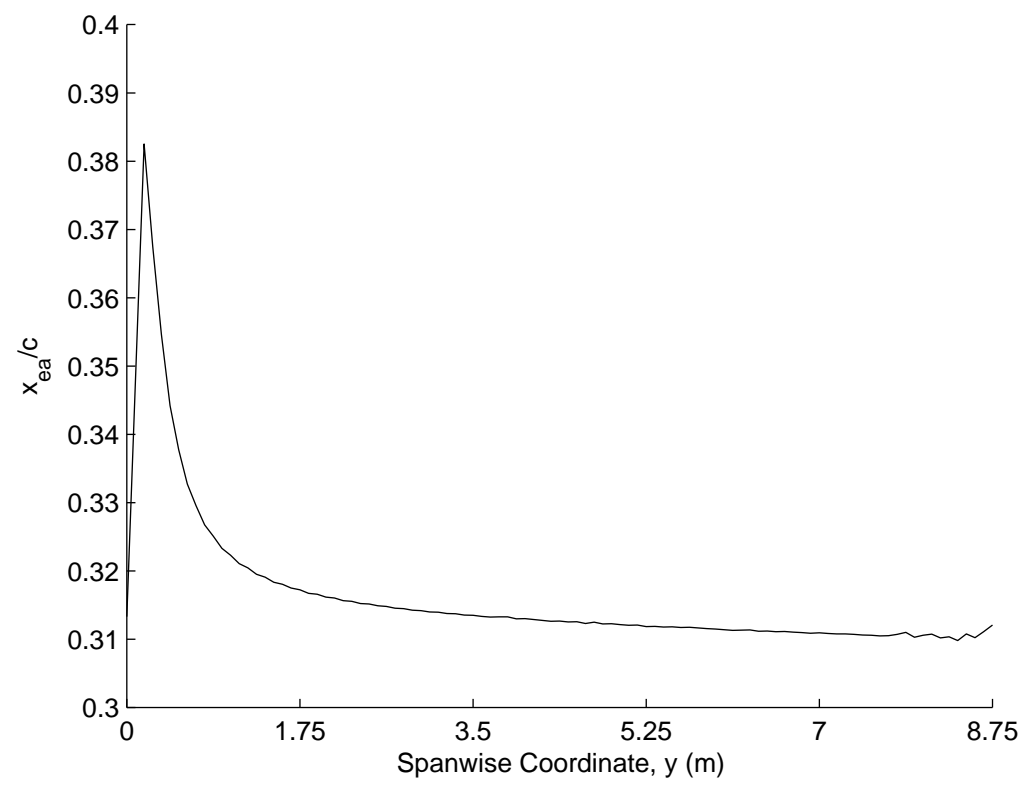

Figure 3.5: Chord-wise location of the elastic axis for the stiff wing as calculated using results from FEA. 


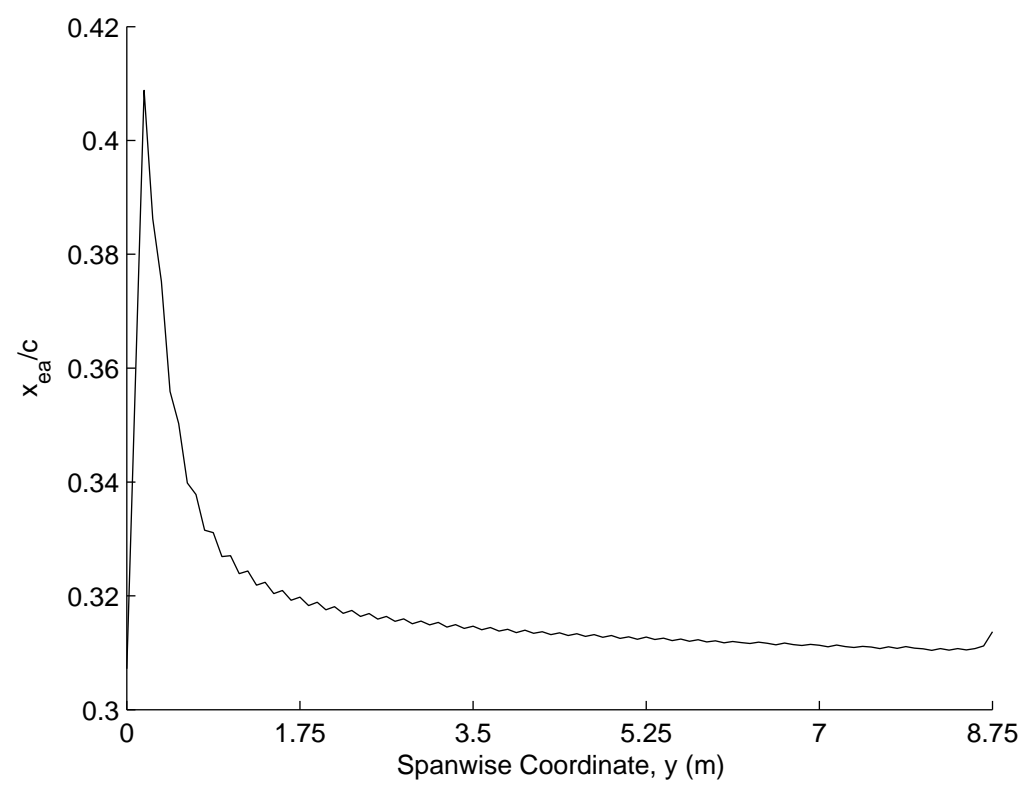

Figure 3.6: Chord-wise location of the elastic axis for the flexible wing as calculated using results from FEA.

are calculated for $C_{L}$ of $0.1,0.5$, and 0.8 and are plotted in Fig. 3.7 and 3.8. For demonstrating the effect of the elasticity, flap angles were also calculated using the rigid-wing methodology of King and Gopalarathnam. ${ }^{7}$ Comparing Fig. 3.7 and Fig. 3.8 shows that wing stiffness can drastically alter the calculated flap angles.

Using both the elastic-wing and rigid-wing flap angles, the $C_{l}$ distribution is calculated for an elastic wing using the methodology presented in this paper. These $C_{l}$ distributions are compared with $C_{l_{\text {optimum }}}$ in Fig. 3.9 and 3.10. For all three wing lift coefficients, the equations presented in this paper produce a $C_{l}$ distribution closer to $C_{l_{\text {optimum }}}$, particularly for $C_{L}=0.1$. At the midpoint of each flap, the $C_{l}$ distributions from the elastic-wing methodology for both wings matches $C_{l_{\text {optimum }}}$ exactly. The methodology to calculate the $C_{l}$ distributions is verified by analyzing the wing with the predicted flap deflections using WINGS and comparing the $C_{l}$ distributions. As shown in Fig. 3.11, the $C_{l}$ distribution from the current method agrees with the post-design analysis from WINGS. 


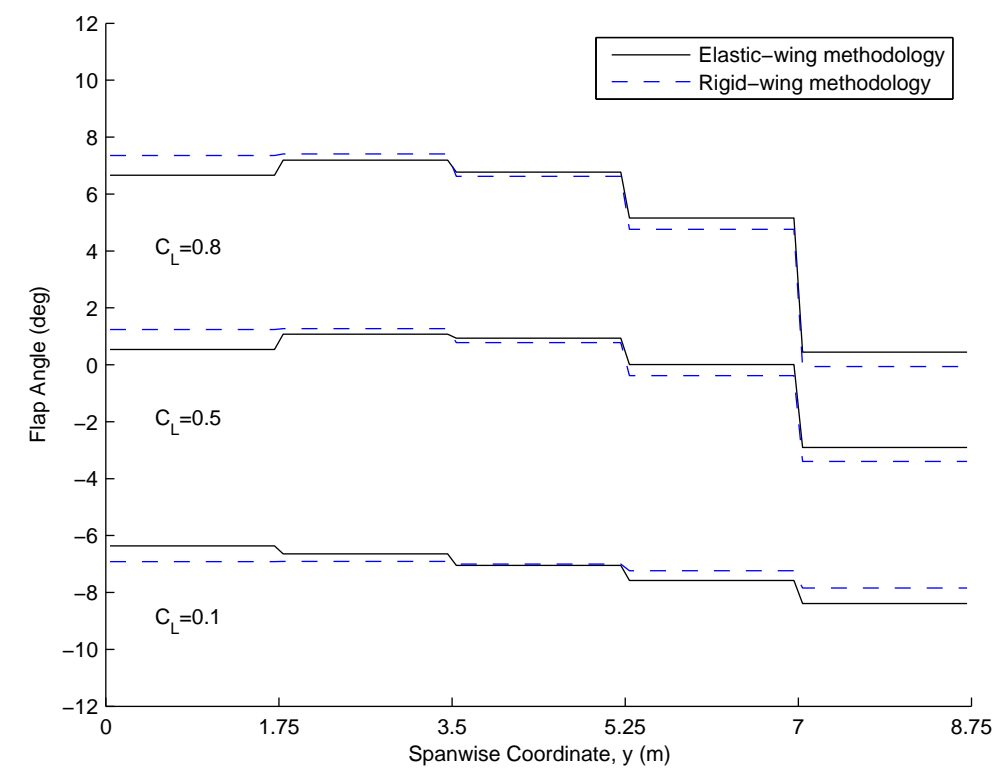

Figure 3.7: Comparison of flap angles for the stiff wing calculated using an elastic wing assumption and a rigid wing assumption for $C_{L}$ of $0.1,0.5$, and 0.8 .

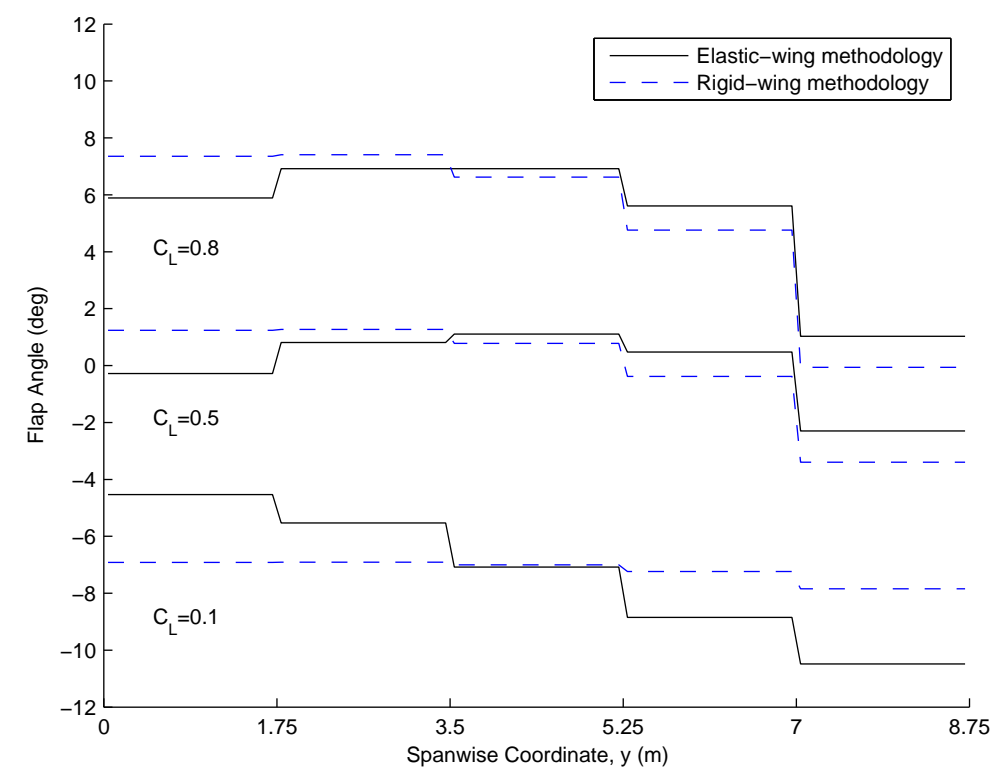

Figure 3.8: Comparison of flap angles for the flexible wing calculated using an elastic wing assumption and a rigid wing assumption for $C_{L}$ of $0.1,0.5$, and 0.8 . 


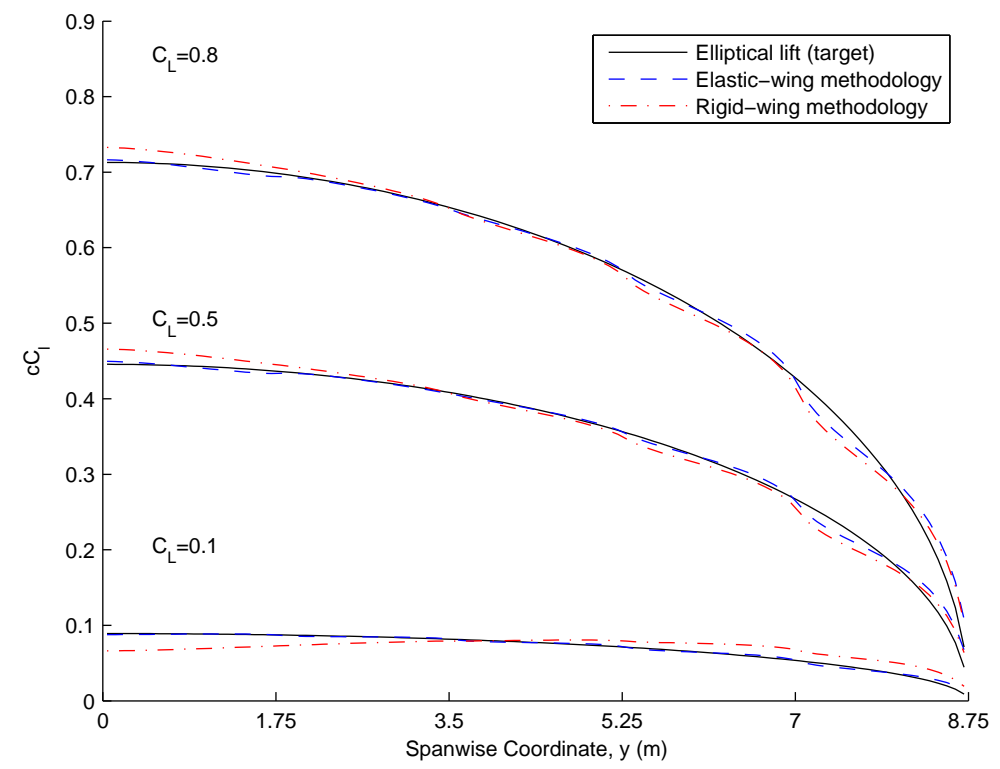

Figure 3.9: Comparison of $C_{l}$ distributions for the stiff wing. The $C_{l}$ distributions are calculated for an elastic wing with flap angles from (i) the elastic-wing methodology and (ii) the rigid-wing method and compared with the optimum $C_{l}$ distribution. 


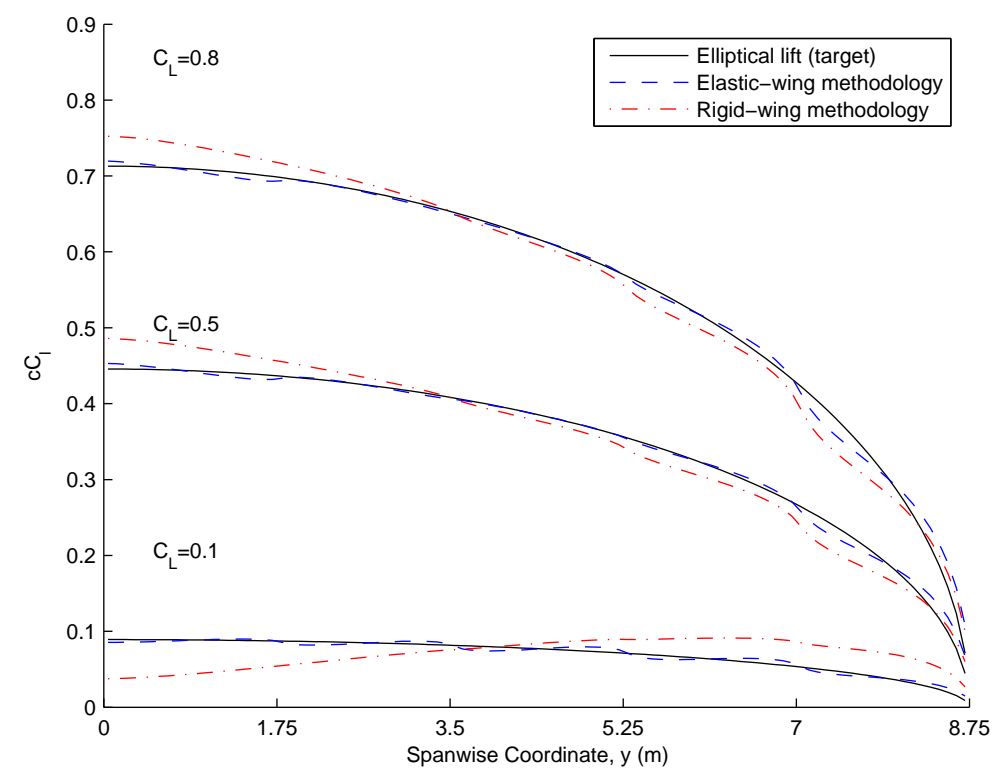

Figure 3.10: Comparison of $C_{l}$ distributions for the flexible wing. The $C_{l}$ distributions are calculated for an elastic wing with flap angles from (i) the elastic-wing methodology and (ii) the rigid-wing method and compared with the optimum $C_{l}$ distribution.

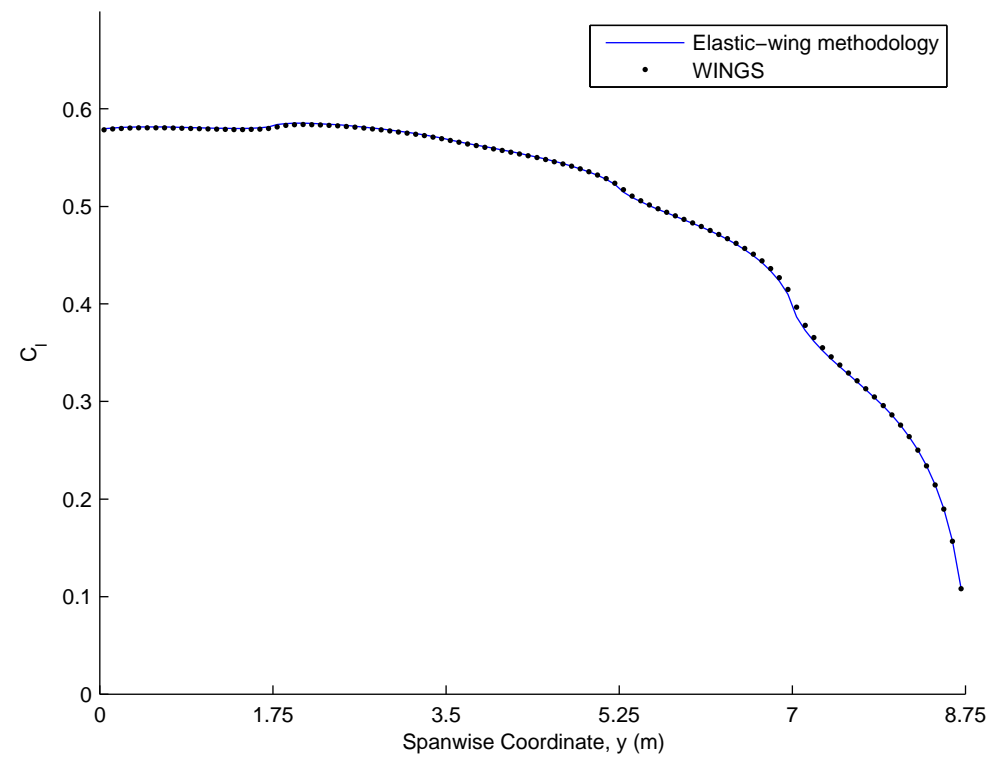

Figure 3.11: Comparison of $C_{l}$ distribution from the current method (Eq. 2.31) with that from post-design analysis using the WINGS code. 
To demonstrate that the wing is still operating within the low-drag range (LDR) of the airfoil, drag polars for the airfoil with the correct flap angles are plotted over the spanwise $C_{l}$ distributions in Fig. 3.12 and 3.13. It is seen that for most cases when using the flap angles predicted by the current elastic-wing methodology, the wing not only achieves a near elliptical lift distribution, but also operates within the LDR over almost the entire span. Thus, both induced drag and profile drag are minimized. The exception is $C_{L}=0.1$ for the flexible wing. In this case, there is a large variation in the flap angles. This result shows that as the flaps deviate further from the mean flap angle, less of the wing will be operating in the LDR. In cases such as $C_{L}=0.1$ for the flexible wing, profile drag is not minimized because very little of the wing is operating within the LDR. In order to increase the percentage of the wing operating in the LDR, a more rigid wing is required.
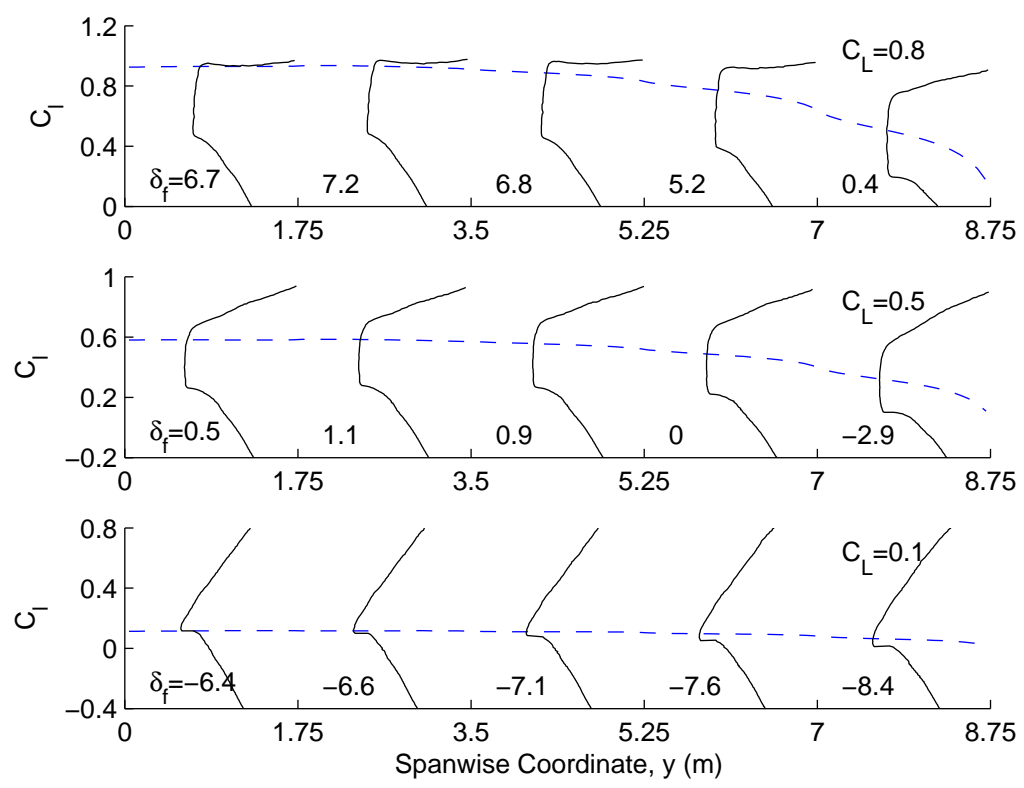

Figure 3.12: $C_{l}$ distributions, flap angles, and drag polars for $C_{L}$ of $0.1,0.5$, and 0.8 for the stiff wing.

Comparing the twist calculated from the current methodology and from FEA 

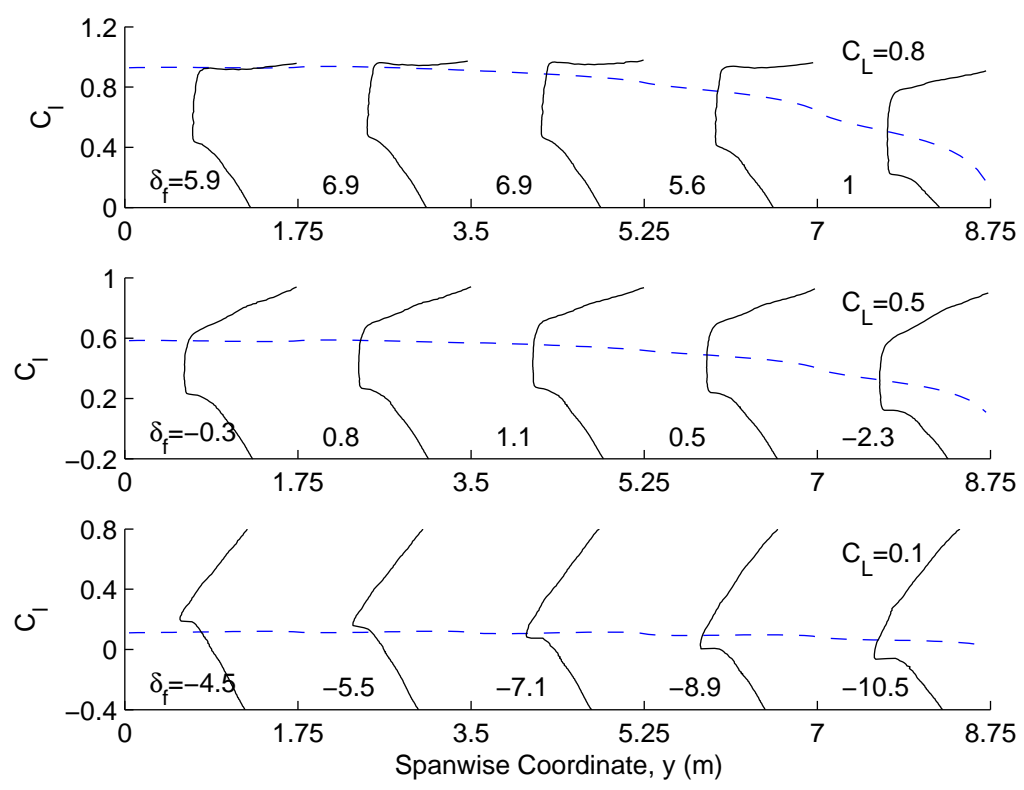

Figure 3.13: $C_{l}$ distributions, flap angles, and drag polars for $C_{L}$ of $0.1,0.5$, and 0.8 for the flexible wing.

verifies the equations (Figs. 3.14 and 3.15). Since the predicted twist and twist from FEA agree well, it can be concluded that the equations correctly predict the aeroelastic effects of the wing.

\subsection{Flap Reversal}

Aileron reversal is the point at which the aileron on the aircraft changes the lift more by twisting the wing than by directly changing the lift coefficient of the section. Since the example wing doesn't have a flap specifically designated as an aileron, the term flap reversal will be used for this wing. From Bisplinghoff et al., ${ }^{13}$ we get the condition for flap reversal (Eq. 3.1). Except for the dynamic pressure, $q_{R}$, all of the variables are constant properties of the wing. Therefore, to demonstrate how flap angles from the current methodology respond to increasing dynamic pressure, only the dynamic pressure needs to be varied. Because of the 

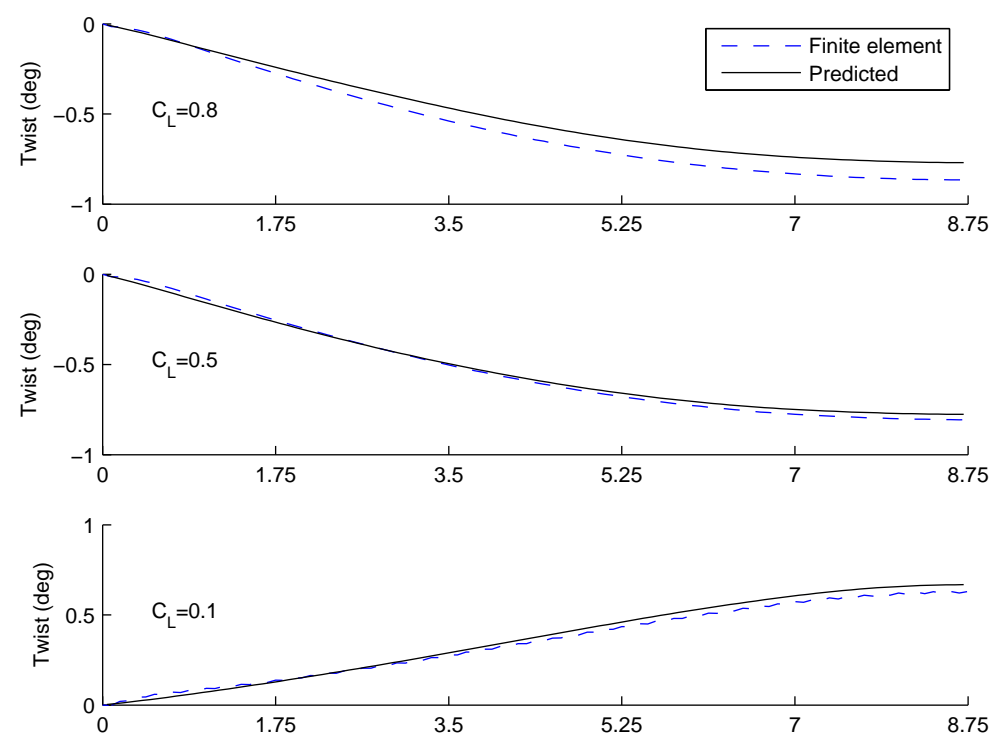

Figure 3.14: Wing twist predicted by current methodology compared to the twist calculated via FEA for $C_{L}$ of $0.1,0.5$, and 0.8 .
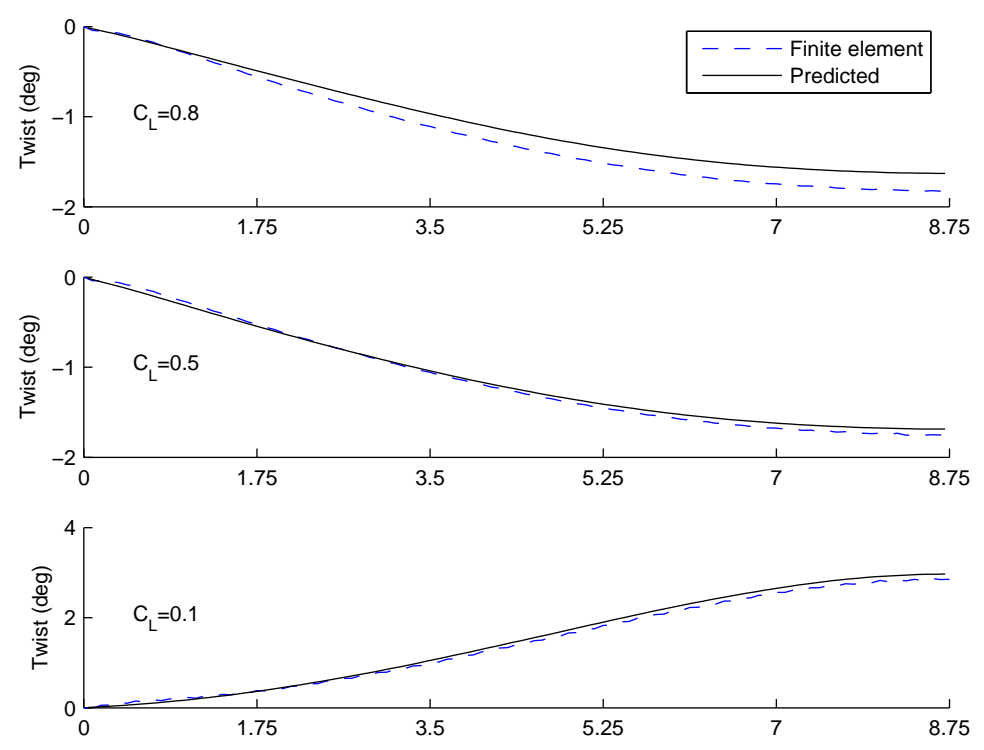

Figure 3.15: Wing twist predicted by current methodology compared to the twist calculated via FEA for $C_{L}$ of $0.1,0.5$, and 0.8 . 


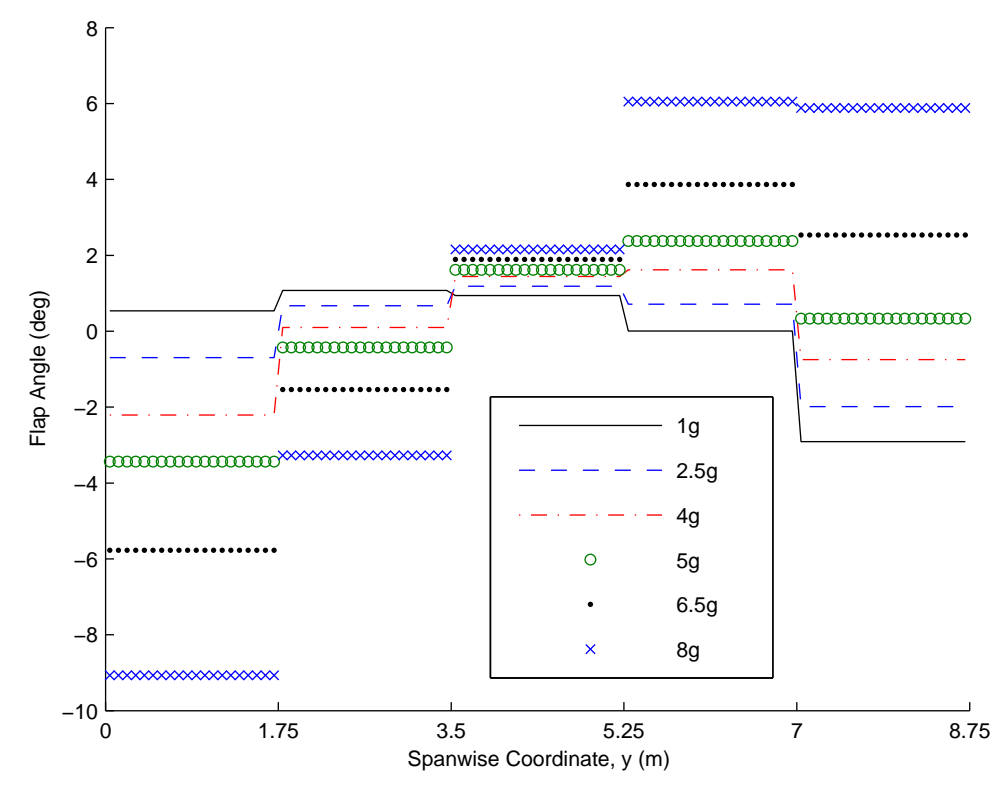

Figure 3.16: Flap angles for $C_{L}$ of 0.5 at increasing loads.

way the current methodology is implemented in code, the easiest way to vary the dynamic pressure is to change the weight of the aircraft while holding $C_{L}$ constant. A $C_{L}$ of 0.5 was chosen so that the mean flap angle would be zero. The properties for the stiff wing are used in the calculations, and the result of increasing the dynamic press on the calculated flap angles is plotted in Fig. 3.16.

$$
\frac{\partial C_{L}}{\partial \delta_{f}} \frac{1}{\left(\partial C_{L} / \partial \alpha\right) C^{\theta \theta} q_{R} S}+c \frac{\partial C_{M A C}}{\partial \delta_{f}}=0
$$

In Fig. 3.17, the $C_{l}$ distributions for $C_{l_{\text {optimal }}}$ and $C_{l_{a}}$ are plotted. For the wing to achieve an elliptical loading, $C_{l}$ needs to be decreased near the tip and increased at the root. At low dynamic pressures (before flap reversal), the flap at the wingtip is deflected upwards to decrease lift. The inboard flaps all have positive flap deflections to increase $C_{l}$. The wing twist (Fig. 3.18) is small, so most of the change in $C_{l}$ is due to flap deflections. At high dynamic pressures, the wing twist is much larger. The increase in twist is caused partially by the 


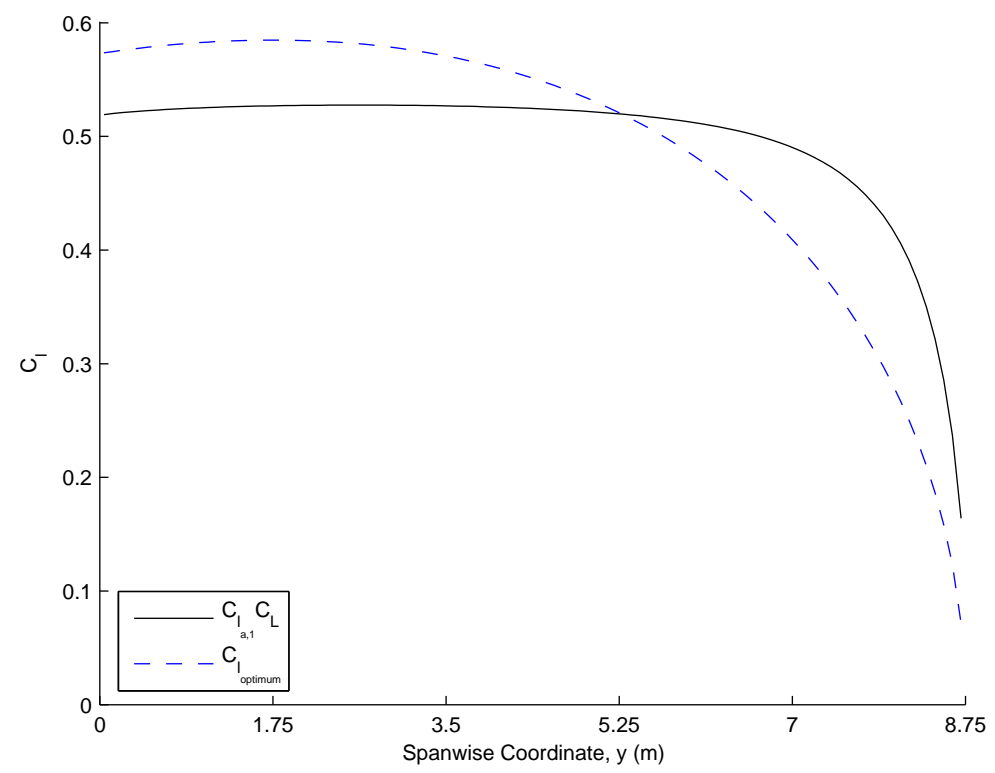

Figure 3.17: $C_{l}$ distributions for $C_{l_{\text {optimum }}}$ and $C_{l_{a}}$ at $C_{L}$ of 0.5 .

positive flap deflections at the wingtip. Since the decrease in lift caused by the twist is greater than the increase in lift caused by the positive flap deflection, the flap at the wingtip is experiencing flap reversal. However near the root, the flaps are not experiencing flap reversal. Because of the decrease in $C_{l}$ near the tip, $C_{l}$ near the root is increased. To counteract the increased lift at the root, the root flap is deflected upwards.

\subsection{Summary}

In this chapter an example was presented to verify the current methodology. The example used a wing with two different skin thicknesses to show the effect of elasticity. The results of the methodology was verified by comparison to results from FEA. A second example was presented to demonstrate flap effectiveness and flap reversal, and their effect upon the flap angles calculated. 


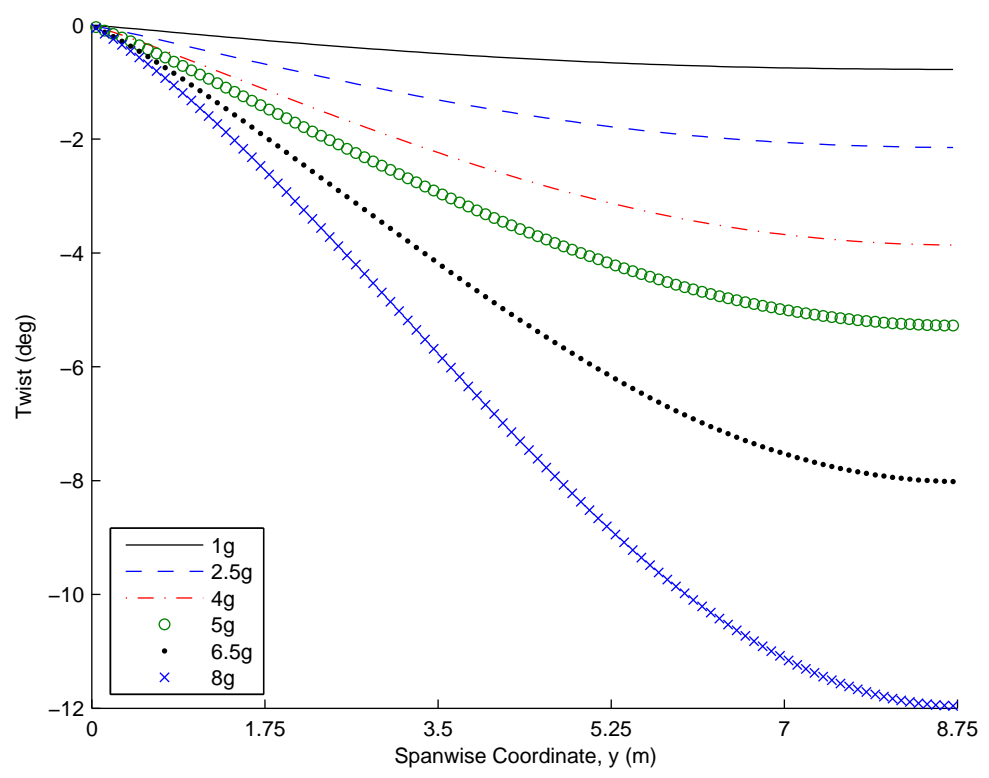

Figure 3.18: Wing twist for $C_{L}$ of 0.5 at increasing loads. 


\section{Chapter 4}

\section{Conclusion}

\subsection{Concluding Remarks}

In this thesis, a methodology has been presented that allows for the calculation of flap angles to reproduce a desired lift distribution and simultaneously minimize profile drag. If the desired lift distribution is elliptical, then the induced drag will also be minimized. This methodology includes calculations for the wing twist and accounts for static aeroelastic phenomena, such as flap reversal and flap effectiveness, in the flap angle calculations.

Examples are then given to verify the current methodology and demonstrate the effect of elasticity. The current methodology is compared to the results of the rigid-wing methodology when applied to an elastic wing. A finite element analysis (FEA) model is used calculate to the wing twist and a post-design analysis code, WINGS, is used to calculate the lift distribution. In both cases the data from FEA and WINGS closely matched the data from the current method, which verifies the methodology.

An example is given to demonstrate flap reversal and flap effectiveness. By changing the dynamic pressure, the effectiveness of flaps at directly changing the $C_{l}$ distribution is reduced and eventually reversed. The flaps at the wing tip experience flap reversal at a lower dynamic pressure than the flaps closer to 
the wing root. The flap at the wing root does not see significant changes in effectiveness due to increased stiffness at the root.

With the inclusion of aeroelasticity into the methodology, the accuracy of the methodology in reproducing a desired $C_{l}$ distribution has been increased. Aircraft with high aspect ratio and very flexible wings will see the largest increase in accuracy. Also the accuracy of the methodology will not diminish with increasing dynamic pressure.

\subsection{Suggestions for Future Research}

In the current methodology, the use of 5 flaps with each flap occupying one-fifth of the half span was arbitrarily chosen. To further refine the methodology, the optimum number of flaps and the distribution of flaps along the half span needs to be studied. If a wing could have an infinite number of flaps, the desired lift distribution could be exactly replicated. However, an infinite number of flaps isn't practical. Therefore, there should exist an optimum number of flaps that balances the increased induced drag from having a finite number of flap against the issues of increased complexity with additional flaps.

Also, from studying the $C_{l}$ distributions, it can be seen that smallest deviation from the desired $C_{l}$ distribution occurs at the root, and the largest deviation occurs at the tip. A more optimum distribution of flaps along the span would have a large flap at the root, and a small flap at the tip. A methodology should be created to determine the optimum number of flaps and distribution of flaps along the wing span. 
Further improvements in accuracy can be achieved by the inclusion of nonlinear aerodynamic and structural effects. Non-linear aerodynamics is the most important, since it will allow for application on aircraft that fly at transonic and supersonic speeds. 


\section{Chapter 5}

\section{References}

1 Thornton, S. V., "Reduction of Structural Loads using Maneuver Load Control in the Advanced Fighter Technology Integration (AFTI)/F-111 Mission Adaptive Wing," NASA TM 4526, September 1993.

2 Bolonkin, A. and Gilyard, G. B., "Estimated Benefits of Variable-Geometry Wing Camber Control for Transport Aircraft," NASA TM 1999-206586, October 1999 .

3 Reich, G. W., Bowman, J. C., and Sanders, B., "Large-Area Aerodynamic Control for High-Altitude Long-Endurance Sensor Platforms," Journal of Aircraft, 2005.

4 Spillman, J. J., "The use of variable camber to reduce drag, weight and costs of transport aircraft," Aeronautical Journal, January 1992, pp. 1-9.

5 Stanewsky, E., "Aerodynamic benefits of adaptive wing technology," Aerospace Science and Technology, Vol. 4, 2000, pp. 439-452.

6 Monner, H. P., Breitbach, E., Bein, T., and Hanselka, H., "Design aspects of the adaptive wing - the elastic trailing edge and the local spoiler bump," The Aeronautical Journal, February 2000, pp. 89-95. 
7 King, R. M. and Gopalarathnam, A., "Ideal Lift Distributions and Flap Angles for Adaptive Wings," AIAA Paper 2004-4722, 22nd AIAA Applied Aerodynamics Conference, Providence, RI, August 2004.

8 Jepson, J. K. and Gopalarathnam, A., "Computational Study of Automated Adaptation of a Wing with Multiple Trailing-Edge Flaps," AIAA Paper 20051035, 43rd AIAA Aerospace Sciences Meeting, Reno, NV, January 2005.

9 Jones, R. T., "The Spanwise Distribution of Lift For Minimum Induced Drag of Wings Having a Given Lift and a Given Bending Moment," NACA TN 2249, December 1950.

10 Powers, S. G. and Webb, L. D., "Flight Wing Surface Pressure and BoundaryLayer Data Report from the F-111 Smooth Variable-Camber Supercritical Mission Adaptive Wing," NASA TM 4789, 1997.

11 Clarke, R., Allen, M. J., Dibley, R. P., Gera, J., and Hodgkinson, J., "Flight Test of the F/A-18 Active Aeroelastic Wing Airplane," NASA TM 213664, August 2005.

12 Wilson, J. R., "A Twisted Approach to Wing Warping," Aerospace America, October 2005, pp. 29-42.

13 Bisplinghoff, R. L., Ashley, H., and Halfman, R. L., Aeroelasticity, Dover Publications, dover ed., 1996.

14 Kuhn, P., "The Initial Torsional Stiffness of Shells with Interior Webs," NACA Technical Note 542, September 1935.

15 Panton, R. L., Incompressible Flow, John Wiley \& Sons, Inc., 2nd ed., 1996.

16 Anderson Jr., J. D., Fundamentals of Aerodynamics, McGraw Hill, 3rd ed., 2001. 
17 Katz, J. and Plotkin, A., Low-Speed Aerodynamics, Cambridge Aerospace Series, Cambridge University Press, Cambridge, UK, 2001.

18 ANSYS Elements Reference, ansys release 9.0 ed., November 2004.

19 Drela, M., XFOIL 6.94 User Guide, December 2001. 